\title{
3 Research Square \\ CXCL13 Correlates with Prognosis, Immune Infiltration, and T Cell Exhaustion in Ovarian Cancer
}

\section{Hailing Duan}

Inner Mongolia Forestry General Hospital (The Second Clinical Medical School of Inner Mongolia, University for the Nationalities)

\section{Ying Lv}

Inner Mongolia Forestry General Hospital (The Second Clinical Medical School of Inner Mongolia, University for the Nationalities)

\section{Pan Liao}

Inner Mongolia Forestry General Hospital (The Second Clinical Medical School of Inner Mongolia, University for the Nationalities)

\section{Yiming Wang}

IMUN: Inner Mongolia University for Nationalities

\section{Zhifang Zheng}

Inner Mongolia Forestry General Hospital (The Second Clinical Medical School of Inner Mongolia, University for the Nationalities)

Honglan Qu ( $\sim$ quhonglan@163.com )

Inner Mongolia Forestry General Hospital https://orcid.org/0000-0001-9397-5584

\section{Research}

Keywords: immune infiltration, immunotherapy, Ovarian cancer, prognosis, T cells exhaustion, CXCL13

Posted Date: November 9th, 2021

DOI: https://doi.org/10.21203/rs.3.rs-1026024/v1

License: (c) (i) This work is licensed under a Creative Commons Attribution 4.0 International License. Read Full License 


\section{Abstract}

Background: CXCL13 is an important chemotactic factor closely related to the biology of cancer cells. The presence work focused on exploring the significance of CXCL13 in prognosis prediction and analyzing the associations of CXCL13 with T cell function and immune infiltration in various cancers, especially ovarian cancer (OV).

Purpose: CXCL13 is associated with prognosis, immune infiltration, and T cell failure of ovarian cancer.

Methods: The Oncomine, GEPIA2 and HPA databases were utilized for analyzing CXCL13 levels within diverse cancers. The significance of CXCL13 in prognosis prediction was explored through Kaplan-Meier Plotter, TCGAportal, and GEPIA2. Meanwhile, the associations of CXCL13 with clinical stage, gene marker sets, and immune infiltration were examined through TISIDB, GEPIA2, and TIMER databases. Besides, CXCL13 was screened to analyze the biological processes (BPs) and KEGGs enriched by co-expression genes. The miRWalk database was employed for analyzing the gene-miRNA interaction network of CXCL13 within OV.

Results: CXCL13 expression decreased in many cancers, which predicted the dismal survival of OV. CXCL13 upregulation was in direct proportion to the increased immune infiltration degrees of many functional T cells (like exhausted T cells) and immune cells. Additionally, some critical genes of exhausted T cells, such as TIM-3, PD-1, LAG3, TIGIT, GZMB, and CXCL13, were closely associated with CXCL13. Moreover, CXCL13 was related to immune response regulatory signaling pathway, leukocyte cell-cell adhesion, cell adhesion molecules (CAMs), and hematopoietic cell lineage.

Conclusion: CXCL13 can serve as a biomarker to predict cancer prognosis, particularly OV. CXCL13 upregulation remarkably elevates the immune infiltration degrees of numerous immune cells, like mast cells, CD8+ T cells, natural killer (NK) cells, and dendritic cells (DCs). Furthermore, CXCL13 is suggested to be closely related to exhausted T cells, which may be used as a candidate regulating factor for T cell exhaustion within OV. Detecting CXCL13 levels contributes to prognosis prediction and CXCL13 regulation within exhausted T cells, which provides a new approach to maximizing the anti-OV efficacy of immunotherapy.

\section{Introduction}

Ovarian cancer (OV) presents a high mortality rate, and the death-to-incidence ratio is impressively high. At present, OV shows no obvious symptoms, and no effective screening approaches are available. As a result, the diagnosis of many OV cases is made in the later stages (FIGO stages III-IV), and the 5-year overall survival (OS) is about $40-45 \%$ [1]. Currently, OV is mainly treated with chemotherapy combined with surgery. The preferred treatment is tailored according to individual features, which usually comprises primary cytoreductive surgery combined with platinumbased chemotherapy and eventual maintenance using bevacizumab (for wild type (WT) BRCA patients or those with HRD sufficiency) or PARP inhibitors (for mutant BRCA cases or those with HRD deficiency) [2]. Notably, immunotherapy has emerged as the standard treatment for different cancers, since it enhances the body immunity against tumor cells. Multiple immune checkpoints can be triggered via ligand-receptor interactions, which may be dysregulated in the tumor for reducing the antitumor response [3]. Therefore, it is urgently needed to illustrate the tumor-immune interaction immunophenotypes and to identify new immune therapeutic targets for OV.

CXCL13, also called BCA-1 (B cell-attracting chemokine 1) or BLC (Blymphocyte chemoattractant), belongs to the homeostatic chemokine. CXCL13 can be produced via stromal cells within the B-cell regions in secondary lymphoid tissues (follicles), like lymph nodes, spleen, Peyer's patches and tonsils [4-8]. CXCL13 level is significantly related 
to human cancer, and its serum expression is used as an early diagnostic and prognostic biomarker for cancer [9]. CXCR5 and/or CXCL13 upregulation predicts the dismal survival of numerous solid tumors [9]. As revealed by many functional articles carried out in the past two decades, CXCR5 and/or CXCL13 expression increases, leading to the excessive activation of related signaling effectors associated with tumor genesis, proliferation, metastasis and survival of cancer cells [10-15]. Many clinical studies have suggested that CXCR5 and/or CXCL13 expression is upregulated, which is related to tumor genesis and the pathogenesis of diverse lymphoma types, including angioimmunoblastic T-cell lymphoma (AITL) [16-18]. Like hematological cancers, the CXCL13/CXCR5 signaling is also utilized by solid tumor cells as the pivotal axis for integrating several cellular cross-talk and intracellular signaling events related to cell proliferation and metastasis [9]. The above results indicate the key function of CXCL13 in tumor genesis, migration, and invasion.

Recent studies report that CXCL13 contributes to immune checkpoint blockade therapy in HGSC [19]. The combined application of CXCL13 and PD-1 blockade therapy can enhance anticancer immunity and suppress cancer proliferation, which is reversed by CD8+ T cell depletion [6]. Additionally, CXCL13 regulates the clustering of B-cells, the secretion of antibodies, and the aggregation of lymphoid within TLSs [20]. CXCL13 upregulation within cancer indicates a more immunoreactive microenvironment, which is likely to predict prolonged patient survival.

The present work conducted a comprehensive analysis on the CXCL13 level and its relation with cancer survival of patients obtained from several databases, including GEPIA2, Oncomine, LOGpc, HPA, Kaplan-Meier plotter, miRWalk, and LinkedOmics. In addition, Tumor Immune Estimation Resource (TIMER) was utilized to analyze the relations between CXCL13 and tumor-infiltrating immune cells (TIICs) within a diverse tumor microenvironment (TME). Results in the present work can help to illustrate the key function of CXCL13 in OV and elucidate the possible relation of CXCL13 with tumor-immune interactions and the underlying mechanism.

\section{Methods}

\section{Analysis based on Oncomine Database}

Oncomine database covers totally 715 gene expression datasets and 86,733 samples, which contributes to data mining [21]. This database was adopted in the present work for assessing the relation of CXCL13 level with cancer prognosis (https://www.oncomine.org/resource/login.html).

\section{Analysis based on GEPIA2 Database}

Gene Expression Profiling Interactive Analysis 2 (GEPIA2) database (http://gepia2.cancer-pku.cn/), an online approach used to interactively analyze gene levels in cancer and non-carcinoma samples obtained from GTEx (Genotype Tissue Expression) and TCGA, provides the customizable functions, such as profile plotting, differential expression, patient survival, correlation, dimensionality reduction and similar gene detection analyses [22]. The association of CXCL13 levels with TCGA-derived cancer survival was examined through "survival analysis". Besides, the association of CXCL13 levels with TIIC gene markers was analyzed by Spearman's correlation analysis in "correlation analysis". Both cancer and non-carcinoma samples were utilized in analyses.

\section{Analysis based on TCGA portal Database}

TCGAportal (http://www.tcgaportal.org) is a web-based portal used to parallelly align several cancers and comprehensively analyze individual cancers. TCGAportal was used to further verify the prognostic value of CXCL13 
expression in cancer patients (http://tumorsurvival.org/) [23]. TCGA covers the pathological and sequencing data for 30 diverse cancers [24].

\section{Analysis based on Kaplan-Meier Plotter Database}

Kaplan-Meier Plotter database has been developed as a web-based approach for the rapid access to the influence of gene expression on 21 cancer survival, as well as 4 great datasets, namely, breast cancer (BC, $n=6234)$, lung cancer ( $L C, n=3452)$, ovarian cancer $(n=2190)$, and gastric cancer $(G C, n=1440)$ [25]. It was adopted in the current work for evaluating the associations of CXCL13 levels with the survival of these 4 cancers. In addition, the pan-cancer and OV datasets were utilized for analyzing CXCK13 levels within diverse OV subtypes. We determined HR (95\% Cls) and log-rank P-values and plotted the survival curves (http://kmplot.com/).

\section{Analysis based on LOGpc Database}

LOGpc, a web server covering numerous datasets that can be used to analyze survival, contains 13 survival terms from 28,098 cancer cases with 26 cancers (such as OSIms, OSkirc and OSblca) and an additional 23 online prognosis prediction approaches [26-33]. Such case samples were mostly obtained from GEO and TCGA databases. LOGpc is publicly accessible and user friendly. The 26 cancer types are divided as 11 system categories based on TCGA. At present, LOGpc only allows for official gene symbol input. After the gene symbol is set by the user and the relevant parameters are selected, the "Kaplan-Meier plot" button is pressed and the results are displayed on the output webpage. For meeting the specific demands of diverse investigators, the clinical confounders are defined for subsequent subgroup analyses. (http://bioinfo.henu.edu.cn/DatabaseList.jsp).

\section{Analysis based on TISIDB Database}

The TISIDB database covers altogether 988 immune-associated anticancer genes, non-carcinoma multi-omics data, molecular profiling data, high-throughput screening (HTS) technologies, and different immunological data resources collected in 7 publicly accessible databases [34]. It allows to analyze the associations between the screened genes and chemokines, lymphocytes, and immunomodulators. The present work employed TISIDB for assessing the association of Annexin levels with OV clinical stages and investigating the relationship of CXCL13 level with immunomodulators and lymphocytes (http://cis.hku.hk/TISIDB).

\section{Analysis based on TIMER Database}

TIMER (Tumor Immune Estimation Resource; cistrome. shinyapps.io/timer), a kind of easy-to-to-use web interface, has provided a computational approach for oncology investigators to comprehensively and dynamically analyze and monitor cancer genomic and immunologic data [35]. It contains gene expression profiling data of 10897 samples covering 32 different kinds of TCGA-derived cancers, which can be used to estimate the 6 TIICs subpopulation abundances, including CD4+ T cells, CD8+ T cells, dendritic cells (DCs), B cells, neutrophils and macrophages. In this study, we adopted constrained least squares fitting for specific gene levels, which showed a negative correlation with the tumor purity of all cancers [36], to predict the 6 TIIC subpopulation abundances. Furthermore, "Gene module" and "Diff Exp module" were utilized for analyzing the CXCL13 level within diverse cancers and the associations of CXCL13 levels with 6 TIIC subpopulation abundances. Wilcoxon test was utilized to assess the significant difference in CXCL13 levels. Statistical significance and purity-adjusted partial Spearman's correlation were adopted to assess the association between CXCL13 levels and immune infiltration. Tumorinfiltration degrees across cancers showing distinct somatic copy number alterations (SCNA) were compared for CXCL13 using "SCNA module" defined by GISTIC 2.0. The module includes high amplification (2), arm-level gain (1), diploid/normal (0), arm-level deletion (-1), and deep deletion (-2). [37] In addition, we utilized "Correlation 
module" to explore the associations of CXCL13 levels with TIIC gene markers selected based on previously published articles, including markers for T cells, B cells, effector T cells, CD8+ T cells, central memory T cells, effector memory T cells, exhausted T cells, resident memory T cells, effector Treg cells, resting Treg cells, neutrophils, T-helper 1 (Th1), dendritic cells (DCs), macrophages, mast cells and natural killer cells (NK cells) [3841]. The scatterplots of CXCL13 gene expression in the specific cancer were drawn by this module based on statistical significance and Spearman's correlation analysis. We displayed gene expression data in the form of log2 RSEM (RNA-Seq by Expectation Maximization).

\section{Analysis based on Human Protein Atlas (HPA) Database}

The HPA database (https://www.proteinatlas.org/) covers gene and pathological data collected from numerous studies conducted using different cell lines and tissue types [42]. This database was adopted in the present work for examining CXCL13 levels within diverse tissues together with CXCL13 mRNA localization in cells.

\section{Analysis based on LinkedOmics Database}

LinkedOmics represents an openly accessible database, which covers multi-omics data of 32 TCGA-derived cancers [43]. Pearson test was conducted for statistical analyses of CXCL13 co-expression by LinkedOmics of "LinkFinder". All data were presented as heat map/volcano map/scatter plots. In addition, we also utilized LinkedOmics of "LinkInterpreter" module for GO annotation (Biological Process, BP), KEGG pathway analysis, and transcription factor-target/miRNA-target/kinase-target enrichment using Gene Set Enrichment Analysis (GSEA) upon the threshold of false discovery rate (FDR)<0.05 for 1000 iterations (http://www.linkedomics.org).

\section{miRWalk Database Analysis}

CXCL13 was imported into the miRWalk2.0 website to screen target gene regulatory miRNAs. MiRNAs discovered through miRWalk tools were enrolled to be possible CXCL13-regulating miRNAs (http://mirwalk.umm.uniheidelberg.de/) [44].

\section{Results}

\section{The mRNA Expression Levels of CXCL13 in Different Types of Human Cancers}

For evaluating CXCL13 levels within cancer and non-carcinoma samples, this study determined CXCL13 levels in diverse cancer and non-carcinoma samples based on Oncomine database. As a result, CXCL13 levels increased in diverse cancers, such as breast cancer (BC), bladder cancer, cervical cancer (CC), leukemia, head and neck cancer (HNC), lymphoma, lung cancer, and OV, in comparison with non-carcinoma samples (Figure 1A). In addition, its expression decreased in kidney cancer, colorectal cancer (CRC), and sarcoma within certain datasets.

Supplementary Table 1 presents more details on CXCL13 levels within diverse cancers.

For evaluating CXCL13 levels within human cancers, this study determined CXCL13 levels based on RNA-seq data from TCGA-derived cancers. Figure 1B presents CXCL13 expression in cancer and non-carcinoma samples derived from TCGA. CXCL13 levels markedly increased (Cervical squamous cell carcinoma and endocervical adenocarcinoma), BRCA (breast invasive carcinoma), DLBC (Lymphoid Neoplasm Diffuse Large B-cell Lymphoma), CESC COAD (colon adenocarcinoma), HNSC (Head and Neck squamous cell carcinoma), ESCA(Esophageal carcinoma), LUAD (Lung adenocarcinoma), KIRC (Kidney renal clear cell carcinoma), OV (Ovarian serous cystadenocarcinoma), LUSC (Lung squamous cell carcinoma), READ (rectum adenocarcinoma), PAAD(Pancreatic 
adenocarcinoma), STAD (stomach adenocarcinoma), SKCM (Skin Cutaneous Melanoma), THYM(Thymoma), TGCT (Testicular Germ Cell Tumors), UCS (Uterine Carcinosarcoma) and UCEC (Uterine Corpus Endometrial Carcinoma) compared with adjacent normal tissues.

\section{Prognostic prediction potential of CXCL13 in cancer}

Based on the results, we exploited the relationship of CXCL13 level with prognosis in four large tumor datasets (ovarian cancer, lung cancer, gastric cancer, and breast cancer) based on the KM Plotter. Decreased CXCL13 level was related to poor prognosis of OV (OS: $P=0.013, \mathrm{HR}=0.85$ [0.75-0.97]; PFS: $\mathrm{P}=0.043, \mathrm{HR}=0.88$ [0.78-1]), lung cancer (OS: $\mathrm{P}=0.048, \mathrm{HR}=0.88$ [0.78-1]; PFS: $\mathrm{P}=0.013, \mathrm{HR}=0.79$ [0.65-0.95]), gastric cancer $(0 S: \mathrm{P}=2.9 \mathrm{e}-07, \mathrm{HR}$ $=0.64$ [0.54-0.76]), and breast cancer (OS: $P=3 e-07, \mathrm{HR}=0.61$ [0.5-0.74]; RFS: $\mathrm{P}=0.11, \mathrm{HR}=0.92$ [0.83-1.02]) (Figure 2A-H). The prognostic significance of CXCL13 was further validated using RNA-seq data from 33 types of malignancies derived from the TCGA portal using GEPIA2 (Figure 2I). For further analysis, poor overall survival (OS) $(P=0.00042, \mathrm{HR}=0.64)$ and disease-free survival $(\mathrm{DFS})(\mathrm{P}=0.017, \mathrm{HR}=0.74)$ (Figure $2 \mathrm{~J}, 2 \mathrm{~K})$ of OV were related to decreased CXCL13 level. Similarly, reduced CXCL13 expression was associated with poorer DFS in BRCA $(P=$ $0.00033, \mathrm{HR}=0.5)$ (Figure $2 \mathrm{~L}), \mathrm{ACC}(\mathrm{P}=0.00099, \mathrm{HR}=0.31)($ Figure $2 \mathrm{M})$ and HNSC $(\mathrm{P}=0.0032, \mathrm{HR}=0.67)($ Figure $2 N)$. However, increased CXCL13 expression was also related to poor DFS in KIRP $(P=0.0034, H R=2.5)(F i g u r e$ 20), KIRC ( $P=0.0066, H R=1.7)$ (Figure $2 P)$, and OS in UVM $(P=0.02, H R=3.2)$ (Figure 2Q). Moreover, CXCL13 expression significantly affected OS in SKCM, KIRC, KIRP, UCEC (Figure S1A-1D). The prognostic significance of CXCL13 was further validated using TCGAportal. CXCL13 expression significantly affected OS in OV, KIRC, UCEC, KIRP, GBM (Figure S1E-1I). 
Table 1

The prognostic potential of CXCL13 in different subtypes of OV by LOGpc.

\begin{tabular}{|c|c|c|c|c|c|c|c|c|}
\hline \multirow[t]{2}{*}{ Variables } & \multicolumn{3}{|c|}{$\begin{array}{l}\text { Univariate } \\
\text { analysis }\end{array}$} & \multicolumn{3}{|c|}{$\begin{array}{l}\text { Multivariate } \\
\text { analysis }\end{array}$} & \multirow[t]{2}{*}{ Prognostic } & \multirow[t]{2}{*}{ Dataset } \\
\hline & P-value & HR & $95 \% \mathrm{Cl}$ & P-value & HR & $95 \% \mathrm{Cl}$ & & \\
\hline TNM & $<0.0001$ & 2.0570 & $\begin{array}{l}1.5460 \\
\tilde{2} \\
2.7380\end{array}$ & $<0.0001$ & 2.0584 & $1.5472 \sim 2.7385$ & Poor/Poor & GSE26193 \\
\hline TNM & 0.0008 & 1.4277 & $\begin{array}{l}1.1600 \\
\tilde{1} \\
1.7580\end{array}$ & 0.0845 & 1.4257 & $0.9529 \sim 2.1329$ & Poor/- & GSE32062 \\
\hline Age & 0.0172 & 1.8910 & $\begin{array}{l}1.1200 \\
\tilde{3} .1940\end{array}$ & 0.0016 & 1.8378 & $1.2598 \sim 2.6810$ & Poor/Poor & GSE49997 \\
\hline Grade & 0.0324 & 0.4585 & $\begin{array}{l}0.2244 \\
\tilde{0} \\
0.9366\end{array}$ & 0.0059 & 0.4881 & $0.2930 \sim 0.8131$ & Good/Good & \\
\hline TNM & 0.0280 & 1.8980 & $\begin{array}{l}1.0720 \\
\tilde{3} \\
3.3620\end{array}$ & 0.0407 & 1.5365 & $1.0184 \sim 2.3181$ & Poor/Poor & \\
\hline Age & 0.1760 & 1.1610 & $\begin{array}{l}0.9353 \\
\tilde{1} \\
1.4410\end{array}$ & 0.0003 & 2.0589 & $1.3873 \sim 3.0558$ & -/Poor & GSE51088 \\
\hline Histology & $<0.0001$ & 2.2070 & $\begin{array}{l}1.5020 \\
\tilde{3} \\
3.2440\end{array}$ & 0.6384 & 0.9413 & $0.7315 \sim 1.2113$ & Poor/- & \\
\hline TNM & 0.0002 & 1.7250 & $\begin{array}{l}1.2910 \\
\tilde{2} \\
2.3040\end{array}$ & 0.0025 & 1.7281 & $1.2124 \sim 2.4632$ & Poor/Poor & \\
\hline Age & 0.1900 & 1.2370 & $\begin{array}{l}0.9001 \\
\tilde{1} \\
1.7010\end{array}$ & 0.4180 & 1.1437 & $0.8264 \sim 1.5827$ & $-/-$ & GSE53963 \\
\hline TNM & 0.0003 & 1.8010 & $\begin{array}{l}1.3060 \\
\tilde{2} \\
2.4820\end{array}$ & 0.0011 & 1.7340 & $1.2467 \sim 2.4117$ & Poor/Poor & \\
\hline SELL & 0.0103 & 0.4333 & $\begin{array}{l}0.2287 \\
\tilde{0} \\
0.8211\end{array}$ & 0.0273 & 0.4816 & $0.2517 \sim 0.9216$ & Good/Good & GSE63885 \\
\hline
\end{tabular}

Note: Bold values indicate $P \otimes 0.05$.

CXCL13 levels predicted the dismal prognosis of OV cases. Therefore, this study further illustrated the mechanism based on LOGpc database for assessing the association of CXCL13 levels with clinicopathological features of patients. Based on univariate analysis and multivariate analysis, CXCL13 level was markedly related to patient TNM stage, age, gender, histology, and grade, except for race (Table 1). In addition, CXCL13 level was related to TNM stage, corresponding to the lymph node metastasis (LNM) degree within OV. As for the association of CXCL13 with OV survival, TNM stage showed the greatest HR GSE9891, HR $=2.4080$ (1.6050 3.6130, $\mathrm{P}<0.0001)$, indicating that CXCL13 level might affect the survival of OV patients by affecting LNM. 


\begin{tabular}{|c|c|c|c|c|c|c|c|c|}
\hline \multirow{2}{*}{$\begin{array}{l}\text { Variables } \\
\text { Grade }\end{array}$} & \multicolumn{3}{|c|}{$\begin{array}{l}\text { Univariate } \\
\text { analysis }\end{array}$} & \multicolumn{3}{|c|}{$\begin{array}{l}\text { Multivariate } \\
\text { analysis }\end{array}$} & \multirow{2}{*}{$\begin{array}{l}\text { Prognostic } \\
\text { Poor/Poor }\end{array}$} & \multirow[t]{2}{*}{ Dataset } \\
\hline & 0.0113 & 1.7070 & $\begin{array}{l}1.1290 \\
\tilde{\tilde{T}} \\
2.5820\end{array}$ & 0.0266 & 1.5954 & $1.0556 \sim 2.4112$ & & \\
\hline TNM & 0.0084 & 2.3150 & $\begin{array}{l}1.2390 \\
\tilde{u} \\
4.3230\end{array}$ & 0.0478 & 1.9292 & $1.0064 \sim 3.6983$ & Poor/Poor & \\
\hline Age & 0.0275 & 1.7860 & $\begin{array}{l}1.0660 \\
\tilde{2} .9930\end{array}$ & 0.1067 & 1.5454 & $0.9106 \sim 2.6228$ & Poor/- & GSE73614 \\
\hline Grade & 0.0068 & 1.8370 & $\begin{array}{l}1.1830 \\
\tilde{2} \\
2.8550\end{array}$ & 0.1358 & 1.4270 & $0.8944 \sim 2.2769$ & Poor/- & \\
\hline TNM & 0.0001 & 1.7300 & $\begin{array}{l}1.3040 \\
\tilde{\tilde{2}} \\
2.2960\end{array}$ & 0.0025 & 1.5673 & $1.1717 \sim 2.0965$ & Poor/Poor & \\
\hline Histotype & 0.0364 & 0.6003 & $\begin{array}{l}0.3722 \\
\tilde{\tilde{0}} \\
0.9682\end{array}$ & 0.1481 & 0.6932 & $0.4219 \sim 1.1390$ & Good/- & GSE8841 \\
\hline Age & 0.1090 & 1.4310 & $\begin{array}{l}0.9237 \\
\tilde{2} \\
2.2170\end{array}$ & 0.1446 & 1.3893 & $0.8932 \sim 2.1611$ & $-/-$ & GSE9891 \\
\hline TNM & $<0.0001$ & 2.4080 & $\begin{array}{l}1.6050 \\
\tilde{3} .6130\end{array}$ & 0.0002 & 2.2841 & $1.4817 \sim 3.5208$ & Poor/Poor & \\
\hline Age & $<0.0001$ & 1.5640 & $\begin{array}{l}1.2600 \\
\tilde{\tilde{T}} \\
1.9410\end{array}$ & 0.0087 & 1.4444 & $1.0977 \sim 1.9008$ & Poor/Poor & TCGA \\
\hline Grade & 0.1680 & 1.2260 & $\begin{array}{l}0.9177 \\
\tilde{\sim} \\
1.6390\end{array}$ & 0.4853 & 1.1580 & $0.7670 \sim 1.7484$ & $-/-$ & \\
\hline Race & 0.0986 & 0.8123 & $\begin{array}{l}0.6347 \\
\tilde{1} \\
1.0400\end{array}$ & 0.0090 & 0.6699 & $0.4960 \sim 0.9049$ & $-/$ Good & \\
\hline TNM & 0.0008 & 1.4280 & $\begin{array}{l}1.1600 \\
\tilde{\sim} \\
1.7580\end{array}$ & 0.0953 & 1.2919 & $0.9562 \sim 1.7453$ & Poor/- & \\
\hline \multicolumn{9}{|c|}{ Note: Bold values indicate $P \otimes 0.05$. } \\
\hline $\begin{array}{l}\text { CXCL13 lev } \\
\text { mechanism } \\
\text { features of } \\
\text { to patient T } \\
\text { related to T } \\
\text { association } \\
3.6130, P<\end{array}$ & $\begin{array}{l}\text { els predicte } \\
\text { based on } \\
\text { oatients. B } \\
\text { VM stage, } \\
\text { VM stage, } \\
\text { of CXCL13 } \\
.0001) \text {, inc }\end{array}$ & $\begin{array}{l}\text { d the dism } \\
\text { OGpc dat } \\
\text { sed on ur } \\
\text { ge, gende } \\
\text { orresponc } \\
\text { with OV } \mathrm{S} \\
\text { icating th. }\end{array}$ & $\begin{array}{l}\text { I progno } \\
\text { pase for } \\
\text { ariate ar } \\
\text { histology } \\
\text { gg to the } \\
\text { vival, Ts } \\
\text { CXCL13 }\end{array}$ & $\begin{array}{l}\text { is of OV c } \\
\text { ssessing } \\
\text { alysis anc } \\
\text { and grad } \\
\text { ymph noc } \\
\text { M stage s } \\
\text { evel migh }\end{array}$ & $\begin{array}{l}\text { es. Ther } \\
\text { e associa } \\
\text { nultivaria } \\
\text { except fo } \\
\text { metastas } \\
\text { wed the } \\
\text { affect the }\end{array}$ & $\begin{array}{l}\text { ore, this study furt } \\
\text { ion of CXCL13 leve } \\
\text { e analysis, CXCL13 } \\
\text { race (Table 1). In } \\
\text { s(LNM) degree wi } \\
\text { reatest HR (GSE98 } \\
\text { survival of OV pati }\end{array}$ & $\begin{array}{l}\text { er illustrated } \\
\text { s with clinico } \\
\text { level was mal } \\
\text { ddition, CXCL } \\
\text { in OV. As for } \\
1 \text {, HR = } 2.408 \\
\text { nts by affecti }\end{array}$ & $\begin{array}{l}\text { e } \\
\text { athological } \\
\text { edly related } \\
3 \text { level was } \\
\text { e } \\
\text { (1.6050 } \\
\text { LNM. }\end{array}$ \\
\hline
\end{tabular}

\section{Regulation of immune molecules by CXCL13}


This study examined Spearman's correlation of CXCL13 levels with immunomodulators and lymphocytes based on TISIDB database (Figure 4). Figure 4A displays the association of CXCL13 levels with tumor-infiltrating lymphocytes (TILs). The most significantly correlated TILs were type 1 T helper cell (Th1; Spearman: $\rho=0.627, P<$ 2.2e-16), Activated B cells (Act-B; Spearman: $\rho=0.682, P<2.2 \mathrm{e}-16$ ), and Activated CD8 T cell (Act-CD8; Spearman: $\rho=0.72, \mathrm{P}<2.2 \mathrm{e}-16)$ (Figure 4B). Additionally, immunomodulators are also divided as major histocompatibility complex (MHC) molecules, immunoinhibitors, and immunostimulators. Figure 4C displays the associations of CXCL13 expression with immunoinhibitors, among which the most significantly correlated ones were CTLA4 (Spearman: $\rho=0.769, \mathrm{P}<2.2 \mathrm{e}-16)$, PDCD1LG2 (Spearman: $\rho=0.731, \mathrm{P}<2.2 \mathrm{e}-16)$, and TIGIT (Spearman: $\rho=$ $0.793, P<2.2 \mathrm{e}-16$ ) (Figure 4D). Figure 4E presents the associations of CXCL13 level with immunostimulators, among which the most significantly correlated ones were CD27 (Spearman: $\rho=0.797, P<2.2 e-16), C D 48$ (Spearman: $\rho=0.714, P<2.2 e^{-16}$ ), and ICOS (Spearman: $\rho=0.81, P<2.2 e-16$ ) (Figure 4F). Figure $4 G$ presents the associations of CXCL13 levels with MHC molecules, among which the most significantly correlated ones were HLAB (Spearman: $\rho=0.59, \mathrm{P}<2.2 \mathrm{e}-16$ ), HLA-F (Spearman: $\rho=0.587, \mathrm{P}<2.2 \mathrm{e}-16$ ), and TAP1 (Spearman: $\rho=0.614, \mathrm{P}<$ 2.2e-16) (Figure 4H). Therefore, CXCL13 might participate in modulating the above-mentioned immune molecules.

\section{CXCL13 correlates with immune infiltration levels in OV}

LNM and survival of cancer cases can be separately estimated based on lymphocyte infiltration degrees within the tumor [45-47]. Therefore, this study further analyzed the association of CXCL13 levels with immune cell infiltration degrees within 39 TIMER-derived cancers (Figure S2). As a result, CXCL13 level was significantly related to tumor

purity within 35 cancers and B cell infiltration degree within 30 cancers. Besides, CXCL13 was related to CD4+T cell infiltration degree within 30 cancers, CD8+T cell infiltration degree within 32 cancers, DC infiltration degree within 32 cancers, neutrophil infiltration degree within 29 cancers, and macrophage infiltration degree within 17 cancers. However, CXCL13 expression was not related to the infiltration degrees of CD8+T cells, CD4+T cells, macrophages, DCs, and neutrophils within Kidney Chromophobe (KICH) (Figure S2P). Whereas in OV, CXCL13 level was markedly related to purity level $(R=-0.432, P=1.87 e-23)$, $B$ cell $(R=0.11, P=1.57 e-02)$, CD8+ T cell $(R=0.339, P=2.19 e-14)$, CD4+T cell $(R=0.308, P=4.88 e-12)$, macrophage $(R=0.058, P=2.01 e-01)$, neutrophil $(R=0.371, P=3.97 e-17)$ and dendritic cell $(R=0.358, P=6.17 \mathrm{e}-16)$ (Figure $5 \mathrm{~A}$ ). Therefore, Kaplan-Meier plots were also drawn based on TIMER database for exploring the association of the infiltration degrees of immune cells with CXCL13 levels within OV. According to our results, CXCL13 level $(P=0.011)$ and DCs $(P=0.039)$ were markedly associated with OV survival (Figure 5B). Deletion or normal copy number across CXCL13 gene locus was related to the higher infiltration degree of immune cells (Figure 5C). This study indicated that CXCL13 exerted a vital part in the immune infiltration degree within OV, especially for DCs. 
Table 2

The association of CXCL13 levels with TIIC gene markers within OV based on TIMER.

\begin{tabular}{|c|c|c|c|c|c|}
\hline \multirow[t]{2}{*}{ Immune cell } & \multirow[t]{2}{*}{ Gene markers } & \multicolumn{2}{|l|}{ None } & \multicolumn{2}{|l|}{ Purity } \\
\hline & & Cor & P-value & Cor & P-value \\
\hline \multirow[t]{2}{*}{ CD8+ T cell } & CD8A & 0.753 & $\star \star \star *$ & 0.752 & $\star \star \star ~$ \\
\hline & CD8B & 0.637 & $\star \star \star$ & 0.637 & $\star \star \star$ \\
\hline \multirow[t]{7}{*}{ T cell } & CD6 & 0.530 & $\star \star \star *$ & 0.559 & $\star \star \star *$ \\
\hline & CD3D & 0.801 & $\star \star \star *$ & 0.814 & $\star \star \star *$ \\
\hline & CD3E & 0.775 & $\star \star \star$ & 0.795 & $\star \star \star$ \\
\hline & SH2D1A & 0.806 & $\star \star \star$ & 0.823 & $\star \star \star$ \\
\hline & TRAT1 & 0.761 & $\star \star \star$ & 0.781 & $\star \star \star$ \\
\hline & CD3G & 0.693 & $\star \star \star$ & 0.684 & $\star \star \star$ \\
\hline & CD2 & 0.802 & $\star \star \star$ & 0.819 & $\star \star \star$ \\
\hline \multirow[t]{9}{*}{ B cell } & BLK & 0.371 & $\star * *$ & 0.364 & $\star \star \star *$ \\
\hline & CD19 & 0.300 & $\star \star \star$ & 0.286 & $\star \star \star$ \\
\hline & FCRL2 & 0.497 & $\star \star \star$ & 0.492 & $\star \star \star$ \\
\hline & MS4A1 & 0.701 & $\star \star \star$ & 0.702 & $\star \star \star$ \\
\hline & KIAA0125 & 0.469 & $\star * \star$ & 0.483 & $\star \star \star$ \\
\hline & TNFRSF17 & 0.641 & $\star \star \star *$ & 0.662 & $\star \star \star *$ \\
\hline & TCL1A & 0.516 & $\star * \star$ & 0.528 & $\star \star \star$ \\
\hline & SPIB & 0.522 & $\star \star \star ~$ & 0.553 & $\star \star \star ~$ \\
\hline & CD79A & 0.689 & $\star \star \star$ & 0.689 & $\star \star \star$ \\
\hline \multirow[t]{2}{*}{ Monocyte } & CD86 & 0.596 & $\star \star \star *$ & 0.600 & $\star \star \star$ \\
\hline & CD115(CSF1R) & 0.420 & $\star * \star$ & 0.455 & $\star \star \star$ \\
\hline \multirow[t]{3}{*}{ TAM } & CCL2 & 0.480 & $\star \star \star ~$ & 0.497 & $\star \star \star ~$ \\
\hline & CD68 & 0.533 & $\star \star \star *$ & 0.559 & $\star \star \star ~$ \\
\hline & IL10 & 0.408 & $\star \star \star$ & 0.406 & $\star \star \star$ \\
\hline \multirow[t]{2}{*}{ M1 Macrophage } & IRF5 & 0.169 & * & 0.208 & ** \\
\hline & COX2(PTGS2) & 0.132 & 0.021 & 0.159 & 0.012 \\
\hline \multirow[t]{2}{*}{ M2 Macrophage } & CD163 & 0.470 & $\star \star \star *$ & 0.494 & $\star \star \star ~$ \\
\hline & VSIG4 & 0.428 & $\star \star \star$ & 0.451 & $\star \star \star$ \\
\hline
\end{tabular}

Note: Cor, Spearman's correlation p-value. Purity, tumor purity-adjusted correlation. None, unadjusted correlation. 


\begin{tabular}{|c|c|c|c|c|c|}
\hline Immune cell & Gene markers & None & & Purity & \\
\hline & MS4A4A & 0.507 & $\star \star \star$ & 0.545 & $\star \star \star$ \\
\hline \multirow[t]{3}{*}{ Mast cell } & TPSAB1 & 0.358 & $\star \star \star$ & 0.373 & $\star \star \star$ \\
\hline & СРАЗ & 0.369 & $\star \star \star$ & 0.381 & $\star \star \star ~$ \\
\hline & MS4A2 & 0.299 & $\star \star \star$ & 0.310 & $\star \star \star$ \\
\hline \multirow[t]{6}{*}{ Neutrophils } & FPR1 & 0.450 & $\star \star \star$ & 0.456 & $\star \star \star ~$ \\
\hline & SIGLEC5 & 0.380 & $\star \star \star$ & 0.407 & $\star \star \star$ \\
\hline & CSF3R & 0.391 & $\star \star \star$ & 0.416 & $\star \star \star$ \\
\hline & FCAR & 0.402 & $\star \star \star$ & 0.399 & $\star \star \star ~$ \\
\hline & FCGR3B & 0.238 & $\star \star \star$ & 0.247 & $\star \star \star$ \\
\hline & CD116(ITGAM) & 0.424 & $\star \star \star$ & 0.465 & $\star \star \star ~$ \\
\hline \multirow[t]{10}{*}{ Natural killer cell } & XCL1 & 0.300 & $\star \star \star$ & 0.311 & $\star \star \star$ \\
\hline & XCL2 & 0.562 & $\star \star \star$ & 0.584 & $\star \star \star$ \\
\hline & NCR1 & 0.399 & $\star \star \star$ & 0.447 & $\star \star \star$ \\
\hline & KIR2DL1 & 0.208 & $\star \star$ & 0.220 & $\star *$ \\
\hline & KIR2DL3 & 0.134 & 0.020 & 0.136 & 0.030 \\
\hline & KIR2DL4 & 0.432 & $\star \star \star$ & 0.448 & $\star \star \star$ \\
\hline & KIR3DL1 & 0.312 & $\star \star \star$ & 0.318 & $\star \star \star$ \\
\hline & KIR3DL2 & 0.227 & $\star \star \star$ & 0.244 & $\star \star \star$ \\
\hline & KIR3DL3 & 0.114 & 0.048 & 0.102 & 0.108 \\
\hline & KIR2DS4 & 0.190 & $\star \star$ & 0.201 & * \\
\hline \multirow[t]{9}{*}{ Dendritic cell } & CCL13 & 0.524 & $\star \star \star$ & 0.560 & $\star \star \star *$ \\
\hline & CD209 & 0.297 & $\star \star \star$ & 0.345 & $\star \star \star$ \\
\hline & HLA-DPB1 & 0.526 & $\star \star \star$ & 0.520 & $\star \star \star$ \\
\hline & HLA-DQB1 & 0.359 & $\star \star \star$ & 0.353 & $\star \star \star$ \\
\hline & HLA-DRA & 0.497 & $\star \star \star$ & 0.478 & $\star \star \star ~$ \\
\hline & HLA-DPA1 & 0.506 & $\star \star \star$ & 0.496 & $\star \star \star$ \\
\hline & BCDA-1(CD1C) & 0.365 & $\star \star \star$ & 0.396 & $\star \star \star$ \\
\hline & BDCA-4(NRP1) & 0.176 & * & 0.205 & * \\
\hline & CD11c(ITGAX) & 0.474 & $\star \star \star$ & 0.510 & $\star \star \star$ \\
\hline
\end{tabular}

Note: Cor, Spearman's correlation p-value. Purity, tumor purity-adjusted correlation. None, unadjusted correlation. 


\begin{tabular}{|c|c|c|c|c|c|}
\hline Immune cell & Gene markers & None & & Purity & \\
\hline \multirow[t]{5}{*}{ Th1 } & T-bet(TBX21) & 0.726 & *** & 0.754 & 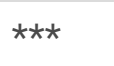 \\
\hline & IFN-Y(IFNG) & 0.771 & 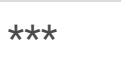 & 0.764 & $\star \star \star$ \\
\hline & TNF-a(TNF) & 0.324 & 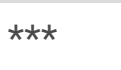 & 0.284 & 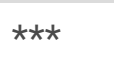 \\
\hline & STAT4 & 0.633 & 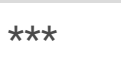 & 0.675 & 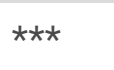 \\
\hline & STAT1 & 0.430 & 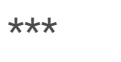 & 0.391 & $\star \star \star ~$ \\
\hline \multirow[t]{2}{*}{ Th2 } & GATA3 & 0.375 & $\star \star \star \star ~$ & 0.393 & $\star \star \star ~$ \\
\hline & STAT5A & 0.156 & * & 0.196 & * \\
\hline Tfh & IL21 & 0.320 & $\star \star \star \star$ & 0.326 & $\star \star \star ~$ \\
\hline \multirow[t]{2}{*}{ Th17 } & STAT3 & 0.180 & * & 0.178 & * \\
\hline & IL17A & 0.152 & 0.008 & 0.165 & 0.009 \\
\hline Effector T cell & FCGR3A & 0.583 & $\star \star \star \star ~$ & 0.587 & $\star \star \star ~$ \\
\hline \multirow[t]{3}{*}{ Effector memory T cell } & PD-1 (PDCD1) & 0.666 & $\star \star \star \star ~$ & 0.716 & $\star \star \star ~$ \\
\hline & GZMK & 0.771 & $\star \star \star \star$ & 0.777 & $\star \star \star$ \\
\hline & GZMA & 0.722 & 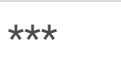 & 0.742 & $\star \star \star ~$ \\
\hline \multirow[t]{3}{*}{ Central memory $\mathrm{T}$ cell } & CCR7 & 0.660 & $\star \star \star \star ~$ & 0.729 & $\star \star \star ~$ \\
\hline & SELL & 0.612 & *** & 0.616 & $\star \star \star ~$ \\
\hline & IL7R & 0.613 & 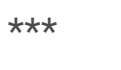 & 0.638 & $\star \star \star ~$ \\
\hline \multirow[t]{2}{*}{ Resident memory $T$ cell } & CD69 & 0.523 & $\star \star \star \star ~$ & 0.542 & $\star \star \star$ \\
\hline & CXCR6 & 0.797 & $\star \star \star \star$ & 0.803 & $\star \star \star ~$ \\
\hline \multirow[t]{5}{*}{ Exhausted T cell } & TIM-3 (HAVCR2) & 0.580 & $\star \star \star \star ~$ & 0.591 & $\star \star \star ~$ \\
\hline & TIGIT & 0.785 & $\star \star \star \star ~$ & 0.795 & $\star \star \star ~$ \\
\hline & LAG3 & 0.637 & *** & 0.619 & $\star \star \star ~$ \\
\hline & GZMB & 0.721 & 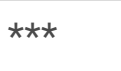 & 0.732 & $\star \star \star ~$ \\
\hline & PD-1 (PDCD1) & 0.666 & *** & 0.716 & $\star \star \star ~$ \\
\hline \multirow[t]{2}{*}{ Resting Treg T cell } & FOXP3 & 0.680 & 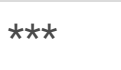 & 0.690 & $\star \star \star ~$ \\
\hline & IL2RA & 0.632 & $\star \star \star \star ~$ & 0.663 & $\star \star \star ~$ \\
\hline \multirow[t]{3}{*}{ Effector Treg T cell } & CTLA4 & 0.764 & $\star \star \star \star ~$ & 0.780 & 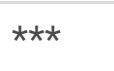 \\
\hline & CCR8 & 0.525 & $\star \star \star \star ~$ & 0.522 & $\star \star \star ~$ \\
\hline & TNFRSF9 & 0.656 & 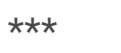 & 0.647 & 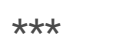 \\
\hline
\end{tabular}


P-value significant codes: * $\mathrm{P}<0.01$; $*$ P $<0.001$; ** $\mathrm{P}<0.0001$.

Page $13 / 33$ 
Table 3

The correlations between CXCL13 and gene markers of immune cells in OV by GEPIA2.

\begin{tabular}{|c|c|c|c|c|c|}
\hline \multirow[t]{2}{*}{ Immune cell } & \multirow[t]{2}{*}{ Gene markers } & \multicolumn{2}{|l|}{ Tumor } & \multicolumn{2}{|c|}{ Tumor-Sum } \\
\hline & & Cor & $P$-value & Cor & $P$-value \\
\hline \multirow[t]{2}{*}{ CD8+ T cell } & CD8A & 0.720 & $\star \star \star \star$ & 0.590 & 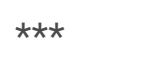 \\
\hline & $\mathrm{CD} 8 \mathrm{~B}$ & 0.066 & 0.170 & & \\
\hline \multirow[t]{7}{*}{$\mathrm{T}$ cell } & CD6 & 0.320 & $\star \star \star$ & 0.660 & 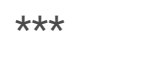 \\
\hline & CD3D & 0.810 & $\star \star \star$ & & \\
\hline & CD3E & 0.750 & $\star \star \star$ & & \\
\hline & SH2D1A & 0.780 & $\star \star \star ~$ & & \\
\hline & TRAT1 & 0.630 & $\star \star \star *$ & & \\
\hline & CD3G & 0.690 & $\star \star \star$ & & \\
\hline & CD2 & 0.760 & $\star \star \star$ & & \\
\hline \multirow[t]{9}{*}{ B cell } & BLK & 0.076 & 0.120 & 0.510 & $\star \star \star$ \\
\hline & CD19 & 0.120 & 0.011 & & \\
\hline & FCRL2 & 0.470 & $\star \star \star$ & & \\
\hline & MS4A1 & 0.420 & $\star \star \star ~$ & & \\
\hline & KIAA0125 & 0.380 & $\star \star \star$ & & \\
\hline & TNFRSF17 & 0.510 & 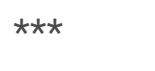 & & \\
\hline & TCL1A & 0.210 & $\star \star \star$ & & \\
\hline & SPIB & 0.290 & $\star \star \star ~$ & & \\
\hline & CD79A & 0.510 & $\star \star \star$ & & \\
\hline \multirow[t]{2}{*}{ Monocyte } & CD86 & 0.440 & $\star \star \star$ & 0.350 & $\star \star \star$ \\
\hline & CD115(CSF1R) & 0.210 & $\star \star \star *$ & & \\
\hline \multirow[t]{3}{*}{ TAM } & CCL2 & 0.150 & $\star \star \star ~$ & 0.330 & $\star \star \star \star ~$ \\
\hline & CD68 & 0.380 & $\star \star \star$ & & \\
\hline & IL10 & 0.170 & $\star \star$ & & \\
\hline \multirow[t]{2}{*}{ M1 Macrophage } & IRF5 & 0.110 & 0.026 & 0.140 & * \\
\hline & COX2(PTGS2) & -0.004 & 0.940 & & \\
\hline \multirow[t]{2}{*}{ M2 Macrophage } & CD163 & 0.300 & 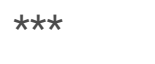 & 0.340 & 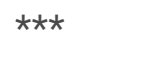 \\
\hline & VSIG4 & 0.200 & $\star \star \star$ & & \\
\hline
\end{tabular}

Notes: Cor, Spearman's correlation p-value. Tumor correlation analysis of single gene markers within OV samples. 


\begin{tabular}{|c|c|c|c|c|c|}
\hline \multirow[t]{2}{*}{ Immune cell } & \multirow{2}{*}{$\begin{array}{l}\text { Gene markers } \\
\text { MS4A4A }\end{array}$} & \multicolumn{2}{|l|}{ Tumor } & \multicolumn{2}{|c|}{ Tumor-Sum } \\
\hline & & 0.350 & $\star \star \star$ & & \\
\hline \multirow[t]{3}{*}{ Mast cell } & TPSAB1 & 0.038 & 0.430 & 0.170 & $\star \star$ \\
\hline & СРАЗ & 0.100 & 0.032 & & \\
\hline & MS4A2 & 0.150 & * & & \\
\hline \multirow[t]{6}{*}{ Neutrophils } & FPR1 & 0.210 & $\star \star \star$ & 0.300 & $\star * \star$ \\
\hline & SIGLEC5 & 0.120 & 0.012 & & \\
\hline & CSF3R & 0.220 & $\star \star *$ & & \\
\hline & FCAR & 0.190 & $\star \star \star$ & & \\
\hline & FCGR3B & 0.150 & * & & \\
\hline & CD116(ITGAM) & 0.230 & $\star \star \star$ & & \\
\hline \multirow[t]{10}{*}{ Natural killer cell } & XCL1 & 0.150 & * & 0.370 & $\star \star \star$ \\
\hline & XCL2 & 0.330 & $\star \star \star$ & & \\
\hline & NCR1 & 0.310 & $\star \star \star$ & & \\
\hline & KIR2DL1 & 0.190 & ** & & \\
\hline & KIR2DL3 & 0.015 & 0.760 & & \\
\hline & KIR2DL4 & 0.270 & $\star \star \star$ & & \\
\hline & KIR3DL1 & 0.230 & $\star \star \star$ & & \\
\hline & KIR3DL2 & 0.260 & $\star \star \star$ & & \\
\hline & KIR3DL3 & 0.099 & 0.041 & & \\
\hline & KIR2DS4 & 0.170 & ** & & \\
\hline \multirow[t]{9}{*}{ Dendritic cell } & CCL13 & 0.270 & $\star \star \star$ & 0.430 & $\star * *$ \\
\hline & CD209 & 0.097 & 0.044 & & \\
\hline & HLA-DPB1 & 0.470 & $\star \star \star ~$ & & \\
\hline & HLA-DQB1 & 0.310 & $\star \star \star$ & & \\
\hline & HLA-DRA & 0.460 & $\star \star \star$ & & \\
\hline & HLA-DPA1 & 0.460 & $\star \star \star$ & & \\
\hline & BCDA-1(CD1C) & 0.140 & * & & \\
\hline & BDCA-4(NRP1) & 0.110 & 0.022 & & \\
\hline & CD11c(ITGAX) & 0.360 & $\star \star \star$ & & \\
\hline
\end{tabular}

Notes: Cor, Spearman's correlation p-value. Tumor correlation analysis of single gene markers within OV samples. 


\begin{tabular}{|c|c|c|c|c|c|}
\hline \multirow{2}{*}{$\begin{array}{l}\text { Immune cell } \\
\text { Th1 }\end{array}$} & \multirow{2}{*}{$\begin{array}{l}\text { Gene markers } \\
\text { T-bet(TBX21) }\end{array}$} & \multicolumn{2}{|l|}{ Tumor } & \multicolumn{2}{|c|}{ Tumor-Sum } \\
\hline & & 0.660 & $\star \star \star$ & 0.490 & $\star \star \star$ \\
\hline & IFN-y(IFNG) & 0.710 & $\star \star \star$ & & \\
\hline & TNF-a(TNF) & 0.140 & * & & \\
\hline & STAT4 & 0.280 & $\star \star \star$ & & \\
\hline & STAT1 & 0.370 & $\star \star \star *$ & & \\
\hline \multirow[t]{2}{*}{ Th2 } & GATA3 & 0.150 & * & 0.200 & $\star \star \star ~$ \\
\hline & STAT5A & 0.130 & * & & \\
\hline Tfh & IL21 & 0.580 & $\star \star \star$ & & \\
\hline \multirow[t]{2}{*}{ Th17 } & STAT3 & 0.200 & $\star \star \star$ & 0.180 & $\star \star$ \\
\hline & IL17A & 0.200 & $\star \star \star$ & & \\
\hline Effector T cell & FCGR3A & 0.400 & $\star \star \star$ & & \\
\hline \multirow[t]{3}{*}{ Effector memory $T$ cell } & PD-1 (PDCD1) & 0.760 & $\star \star \star$ & 0.610 & $\star \star \star$ \\
\hline & GZMK & 0.570 & $\star \star \star$ & & \\
\hline & GZMA & 0.660 & $\star \star \star$ & & \\
\hline \multirow[t]{3}{*}{ Central memory $\mathrm{T}$ cell } & CCR7 & 0.480 & $\star \star \star$ & 0.530 & $\star \star \star$ \\
\hline & SELL & 0.440 & $\star \star \star$ & & \\
\hline & IL7R & 0.370 & $\star \star \star$ & & \\
\hline \multirow[t]{2}{*}{ Resident memory $\mathrm{T}$ cell } & CD69 & 0.210 & $\star \star \star$ & 0.390 & $\star * *$ \\
\hline & CXCR6 & 0.760 & $\star \star \star$ & & \\
\hline \multirow[t]{5}{*}{ Exhausted T cell } & TIM-3 (HAVCR2) & 0.470 & $\star \star \star \star ~$ & 0.680 & $\star \star \star *$ \\
\hline & TIGIT & 0.780 & $\star \star \star$ & & \\
\hline & LAG3 & 0.540 & $\star \star \star$ & & \\
\hline & GZMB & 0.550 & 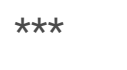 & & \\
\hline & PD-1 (PDCD1) & 0.760 & $\star \star * *$ & & \\
\hline \multirow[t]{2}{*}{ Resting Treg T cell } & FOXP3 & 0.540 & $\star \star \star$ & 0.530 & $\star * *$ \\
\hline & IL2RA & 0.470 & $\star \star \star$ & & \\
\hline \multirow[t]{3}{*}{ Effector Treg T cell } & CTLA4 & 0.750 & $\star \star \star$ & 0.570 & $\star \star \star$ \\
\hline & CCR8 & 0.038 & 0.430 & & \\
\hline & TNFRSF9 & 0.520 & $\star \star \star$ & & \\
\hline $\begin{array}{l}\text { Notes: Cor, Spearman's c } \\
\text { samples. }\end{array}$ & $p$-value. Tumo & tion ar & of $\sin$ & e mar & \\
\hline
\end{tabular}


Tumor-Sum correlation analysis with gene markers set within OV samples.

OV P-value significant codes: * $\mathrm{P}<0.01$; ** $\mathrm{P}<0.001$; ** $\mathrm{P}<0.0001$.

\section{Correlation between CXCL13 and gene markers of immune cells}

To reveal the underlying associations of CXCL13 expression with immune infiltration degree, we analyzed the associations of CXCL13 level with TIIC gene markers in OV based on GEPIA2 and TIMER.

Based on prior studies, the current work adopted the commonly used TIIC gene markers and diverse functional T cells. Table 2 presents the tumor purity-adjusted results of correlation analysis for OV. Clearly, CXCL13 was markedly related to gene markers of monocytes, B cells, TAMs, CD8+ T cells, neutrophils, T cells, DCs, mast cells, NK cells, and many functional T cells. It was interesting that such observations verified that CXCL13 was related to

T cells, B cells, as well as functional T cells reported previously, and illustrated the tight relationship of CXCL13 with mast cells.

For confirming the above observations, this study also examined the correlations of CXCL13 levels with TIIC gene markers within OV and non-carcinoma tissues using GEPIA2 (Table 3). Likewise, CXCL13 shows positive relationships to $T$ cell (Cor $=0.66, P<0.0001)$, CD8+ T cells (Cor $=0.59, P<0.0001)$, $B$ cells $($ Cor $=0.51, P<0.0001)$, monocytes (Cor $=0.35, \mathrm{P}<0.0001)$, TAMs (Cor $=0.33, \mathrm{P}<0.0001), \mathrm{M} 2$ macrophages $($ Cor $=0.34, \mathrm{P}<0.0001)$, neutrophils (Cor $=0.30, \mathrm{P}<0.0001)$, dendritic cells (Cor $=0.43, \mathrm{P}<0.0001)$, natural killer cells $($ Cor $=0.37, \mathrm{P}<$ 0.0001), and multiple functional T cells, especially effector memory T cells (Cor $=0.61, P<0.0001)$, central memory T cells (Cor $=0.53, \mathrm{P}<0.0001)$, resident memory T cells (Cor $=0.39, \mathrm{P}<0.0001)$, exhausted T cells $($ Cor $=0.68, \mathrm{P}<$ 0.0001), resting Treg T cells (Cor $=0.53, \mathrm{P}<0.0001)$, effector Treg T cells (Cor $=0.57, \mathrm{P}<0.0001)$, Th1 cells (Cor $=$ $0.49, \mathrm{P}<0.0001)$ and Th2 cells (Cor $=0.20, \mathrm{P}<0.0001)$. Additionally, increased CXCL13 level was also related to the higher DC infiltration degree within OV. Consistently, DC markers CD11c, HLA-DPB1, CCL13, HLA-DQB1, HLA-DRA, BDCA-1, and HLA-DPA1 were related to CXCL13 level, suggesting the critical role of CXCL13 in tumor DC infiltration. DCs can elevate tumor metastasis degree through promoting the response of Tregs while inhibiting cytotoxicity of CD8+ T cells [48]. More investigations are needed to examine the function of CXCL13 in modulating DC tumor metastasis and infiltration. According to the obtained results, CXCL13 was significantly related to several critical genes related to exhausted T cells, including TIM-3 (Cor $=0.47, \mathrm{P}<0.0001$ ), PD-1 (Cor $=076, \mathrm{P}<.0001$ ), GZMB (Cor $=0.55, \mathrm{P}<0.0001)$, LAG3 (Cor $=0.54, \mathrm{P}<0.0001)$, and TIGIT (Cor $=0.78, \mathrm{P}<.0001)$. They exerted important effects on the existing antitumor immunotherapy.

\section{Expression Levels of CXCL13 in OV}

CXCL13 expression was detected in 4 types including C1 (wound healing), C2 [interferon Y (IFN-y) dominance], C3 (inflammation), and C4 (lymphocyte depletion) types. The highest and lowest CXCL13 levels were detected in C2 and C3 types, respectively (Figure 5A). This study also detected CXCL13 levels within diverse OV molecular subtypes in TISIDB. There are 4 molecular subtypes detected in OV [49], including immunoreactive, mesenchymal, proliferative, and differentiated. As discovered by TISIDB, the greatest and lowest CXCL13 levels were detected in immunoreactive and proliferative subtypes, respectively (Figure 5B), suggesting the close relationship between CXCL13 expression and tumor immune microenvironment (TIME). Similarly, the comparison of different OV stages

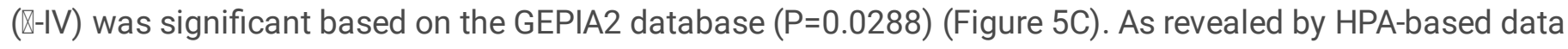
analysis, intense CXCL13 staining was detected in OV samples relative to non-carcinoma ovarian tissues (Figure 5D). 


\section{CXCL13 co-expression network in OV}

To further understand CXCL13's biological significance in OV, the LinkedOmics of "LinkFinder" module was adopted for checking the CXCL13 co-expression patterns. Figure 6A revealed that, altogether 8577 genes (red dots) showed positive correlation with CXCL13, whereas 11433 (green dots) presented a negative correlation $(p<0.05)$. Figures 6B and 6C display the heatmaps for the 50 most significant CXCL13-related genes (both positive and negative). According to the GSEA-annotated GO terms, CXCL13 co-expression genes were mostly associated with leukocyte differentiation, leukocyte cell-cell adhesion, response to a molecule of bacterial origin, immune response-regulating signal transduction pathway, and the regulation of immune effector process. In contrast, it is not involved in cytoskeleton-dependent intracellular transport, ribonucleoprotein complex subunit organization, and microtubule bundle formation (Figure 6D). KEGG analysis revealed that the genes were mostly related to osteoclast differentiation, cell adhesion molecules (CAMs), cytokine-cytokine receptor interaction, chemokine signaling pathway, hematopoietic cell lineage, natural killer cell-mediated cytotoxicity, NK-kappa B signaling pathway, and phagosome (Figure 6E).

\section{miRNA screening of regulatory CXCL13}

miRWalk was applied to screen the targeted miRNAs of CXCL13. Then, miRWalk was used to draw the miRna gene network. As illustrated in Figure 6F, the interaction network consists of CXCL13 and 191 miRNAs. Moreover, the contribution level of miRNAs to CXCL13 is presented as the number of lines. Additionally, the top 20 miRNAs targeting CXCL13 are presented in Figure 6F.

\section{Discussion}

CXCL13, a kind of homeostasis chemokine, was originally called BCA-1 or BLC. CXCL13 participates in tumor genesis, proliferation, metastasis and survival of cancer cells[4-8]. However, its relations with T cell function, immune infiltration, and prognosis of diverse cancers remain unclear. Therefore, this study examined cancer samples from multiple databases for analysis. As a result, CXCL13 expression was related to the prognosis of different cancers, in particular OV. Moreover, CXCL13 co-expression genes also have significant prognostic significance in ovarian cancer. CXCL13 levels revealed a positive correlation with immune infiltration degree within OV. After examining the associations of gene levels among diverse T cells, CXCL13 was confirmed to significantly relate to many functional T cells within OV, especially exhausted T cells. Therefore, CXCL13 might serve as a candidate prognostic biomarker for OV, which offers a new direction to analyze the associations of CXCL13 expression with $\mathrm{T}$ cell function and immune infiltration degree.

The present work analyzed CXCL13 expression with systematic prognosis of diverse cancers based on separate datasets from Oncomine and 33 TCGA-derived cancers from GEPIA2. CXCL13 was differentially expressed between tumor and non-carcinoma samples in diverse cancers. According to Oncomine database-based analysis, CXCL13 expression increased in Bladder cancer, BC, CC, HNC, lymphoma, leukemia, OV, and lung cancer compared to normal tissues, whereas certain datasets indicated that CXCL13 was lowly expressed within CRC, kidney cancer and sarcoma (Figure 1A). However, TCGA-based data analysis revealed that CXCL13 was upregulated in BRCA, CESC, COAD, DLBC, ESCA, HNSC, KIRC, LUAD, LUSC, OV, PAAD, READ, SKCM, STAD, TGCT, THYM, UCEC, and UCS, compared with normal adjacent tissues (Figure 1B). Human Protein Atlas data also verified that CXCL13 expression increased within ovarian cancer, as suggested by Immunohistochemistry (Figure 5D). 
The different CXCL13 levels within diverse cancers from diverse databases might reflect different data extraction methods and biological properties. Based on Kaplan-Meier Plotter and GEPIA2 data analysis, CXCL13 downregulation predicted the dismal survival of OV, BRCA, ACC, and HNSC (Figure 2). In addition, based on univariate analysis and multivariate analysis, CXCL13 expression was significantly correlated with TNM, stage of the patient, age, gender, histology, and grade, except for race. CXCL13 level was related to TNM stage, corresponding to LNM degree within OV, and TNM stage exhibited the highest HR (Table 1). Collectively, the above results indicated that CXCL13 might serve as a prognostic marker for OV.

The present work evaluated the association of CXCL13 levels with the immune system based on TISIDB database. According to our findings, CXCL13 was the most significantly related to lymphocytes (including Th1, Act-B, and ActCD8), immunoinhibitors (such as CTLA4, PDCD1LG2, and TIGIT), MHC molecules (like HLA-B, TAP1, HLA-F), and immunostimulators (such as CD27, CD48, and ICOS). Epigenetic silencing of T1-type chemokines can be a new immune escape mechanism in cancer, while selective epigenetic reprogramming promotes the anti-OV therapeutic effect [50]. Membrane-bound PD-L1 has been the most significant OV biomarker over the last decade, which is induced by TAMs-derived soluble inflammatory factors, resulting in immune invasion [51]. Simultaneous blocking of PD-1-PD-L1 and CXCL12- CXCR4 pathways can suppress OV proliferation and avoid immunosuppression [52]. Besides, osteopontin upregulation can increase PD-L1 levels within hepatocellular carcinoma (HCC) cells via the activation of CSF1-CSF1R pathway within macrophages, whereas blocking CSF1/CSF1R avoids TAM tracking. Therefore, CSF1R inhibitors may be used to promote the PD-L1 antibody efficacy together [53]. Consequently, CXCL13, which is related to the above immune molecules, offers a novel target to study immune escape in OV, which can be used to be the immunotherapeutic target for OV.

However, OV is by no means a single disorder, which is further classified as numerous molecular subtypes. According to TISIDB database-based analysis, CXCL13 gene displayed the greatest expression within the immunoreactive subtype, while that within the mesenchymal type ranked the second place, and CXCL13 was lowly expressed within differentiated and proliferative types. Differential CXCL13 expression within OV of diverse immune subtypes was detected. The results suggested that the $\mathrm{C} 2$ displayed the greatest expression relative to that in the remaining 3 subtypes. The integrative analysis of CXCL13 gene levels across OV and diverse subtypes from various databases possibly suggests that CXCL13 is closely associated with the immune characteristics in TME.

Given that CXCL13 has an important effect on the immune system and on predicting the prognosis of OV, this study examined the association of CXCL13 with immune infiltration degree within OV (Figure 4A). As a result, CXCL13 upregulation was closely associated with the infiltration degrees of many immune cells, like $B$ cells, CD4+T cells and especially, CD8+ T cells, dendritic cells and neutrophils, which have a stronger correlation levels. Additionally, DC infiltration was significantly correlated with OV prognosis (Figure 4B). The diverse SCNA of CXCL13 did not significantly affect the macrophage immune infiltration degrees within OV (Figure 4C), and we paid attention to the relationship of CXCL13 with immune cells.

As suggested by subsequent analyses on the relationships of CXCL13 with TIIC gene markers, CXCL13 interacted with many immune cells and diverse functional T cells, including central memory $\mathrm{T}$ cells, effector $\mathrm{T}$ cells, and exhausted T cells (Tables 2 and 3). Since T cell exhaustion accounts for a leading reason for the ineffective anticancer immunity [54-56], the measures for preventing T cell exhaustion represent the keys to anticancer immunotherapy. Based on our results, CXCL13 upregulation showed a positive correlation with several critical genes related to exhausted T cells, such as TIM-3, PD-1, LAG3, TIGIT, and GZMB. These are therapeutic targets for immunotherapy $[57,58]$. 
According to our findings, CXCL13 exerts dual functions, where its upregulation shows a positive correlation with favorable survival of some cancers including OV. In the meantime, it can induce T cell exhaustion that may induce inefficient anticancer immunity. Consequently, CXCL13 has important yet different functions in normal immune development and in the regulation of TME, which deserves further investigation.

This study identified that CXCL13 was related to mast cells within OV, which has not been reported previously. Mast cells exert the effector activity in the case of TH2-skewed autoimmune and allergic inflammation, enhance sufficient inflammatory responses, and activate T cell in cooperation with DCs [59]. Some recent studies suggest that mast cells do significantly affect TME conformation or promote cancer development [60, 61]. In our study, CXCL13 upregulation indicated higher levels of certain critical gene markers (TPSAB1, CPA3, MS4A2) in mast cells, implying that CXCL13 had an important function. Moreover, more studies are needed to examine the related mechanism.

\section{Conclusions}

Taken together, results in the current work indicate the potential of CXCL13 as a prognostic biomarker for several cancers, in particular OV. CXCL13 upregulation is related to the greater immune infiltration degree of neutrophils, DCs, B cells, CD4+T cells, CD8+T cells, and many functional T cells. CXCL13 generates an important effects on immunity and it shows high correlation with exhausted T cells, which may serve as an important factor to promote $T$ cell exhaustion within OV. The detection of CXCL13 levels possibly contributes to prognosis prediction. Besides, modulating CXCL13 levels within exhausted T cells is a novel approach to optimizing the efficacy of anti-OV immunotherapy.

\section{Abbreviations}

OV

Ovarian cancer

AITL

angioimmunoblastic T-cell lymphoma

CAM

cell adhesion molecules

NK

Natural killer

DCs

dendritic cells

OS

overall survival

DFS

disease-free survival

BCA-1

B cell-attracting chemokine 1

BLC

Blymphocyte chemoattractant

TIIC

tumor-infiltrating immune cells 
TME

tumor microenvironment

GEPIA2

Gene Expression Profiling Interactive Analysis 2

GTEx

Genotype Tissue Expression

HTS

high-throughput screening

TIMER

Tumor Immune Estimation Resource

SCNA

somatic copy number alteration

Th1

T-helper 1

RSEM

RNA-Seq by Expectation Maximization

HPA

Human Protein Atlas

GSEA

Gene Set Enrichment Analysis

$\mathrm{BC}$

breast cancer

cC

cervical cancer

$\mathrm{HNC}$

head and neck cancer

CRC

colorectal cancer

BRCA

breast invasive carcinoma)

DLBC

Lymphoid Neoplasm Diffuse Large B-cell Lymphoma

CESC

Cervical squamous cell carcinoma and endocervical adenocarcinoma

COAD

colon adenocarcinoma

HNSC

Head and Neck squamous cell carcinoma

ESCA

Esophageal carcinoma

LUAD

Lung adenocarcinoma

KIRC

Kidney renal clear cell carcinoma 
LUSC

Lung squamous cell carcinoma

READ

rectum adenocarcinoma

PAAD

Pancreatic adenocarcinoma

STAD

stomach adenocarcinoma

SKCM

Skin Cutaneous Melanoma

THYM

Thymoma

TGCT

Testicular Germ Cell Tumors

UCS

Uterine Carcinosarcoma

UCEC

Uterine Corpus Endometrial Carcinoma

KM

Kaplan-Meier

UVM

Uveal Melanoma

GBM

Glioblastoma multiforme

KIRP

Kidney, renal, papillary cell carcinoma

LNM

lymph node metastasis

TILs

tumor-infiltrating lymphocytes

$\mathrm{MHC}$

major histocompatibility complex

$\mathrm{KICH}$

Kidney Chromophobe

TIME

tumor, immune microenvironment

KEGG

Kyoto Encyclopedia of Genes and Genomes

ACC

Adrenocortical carcinoma

$\mathrm{HCC}$

hepatocellular carcinoma

Declarations 


\section{Ethics approval and consent to participate}

TCGA and GEO belong to public databases. The patients involved in the database have obtained ethical approval. Users can download relevant data for free for research and publish relevant articles. Our study is based on open source data, so there are no ethical issues and other conflicts of interest.

\section{Consent for publication}

Not applicable.

\section{Availability of data and materials}

Data supporting our findings are already included in the manuscript.

\section{Competing interests}

The authors declare that they have no competing interests.

\section{Funding}

Not applicable.

\section{Authors' contributions}

(I) Conception and design: Honglan Qu; (II) Administrative support: All authors; (III) Provision of study materials or patients: None; (IV) Collection and assembly of data: None; (V) Data analysis and interpretation: All authors; (VI) Manuscript writing: Hailing Duan and Ying Lv; (VII) Final approval of manuscript: All authors.

\section{Acknowledgements}

We acknowledge TCGA and GEO database for providing their platforms and contributors for uploading their meaningful datasets.

\section{References}

1. Sambandam Y, Sundaram K, Liu A, Kirkwood KL, Ries WL, Reddy SV. CXCL13 activation of c-Myc induces RANK ligand expression in stromal/preosteoblast cells in the oral squamous cell carcinoma tumor-bone microenvironment. Oncogene. 2013;32:97-105.

2. Henderson JT, Webber EM, Sawaya GF. Screening for Ovarian Cancer: Updated Evidence Report and Systematic Review for the US Preventive Services Task Force. Jama. 2018;319:595-606.

3. Takaya H, Nakai H, Takamatsu S, Mandai M, Matsumura N. Homologous recombination deficiency statusbased classification of high-grade serous ovarian carcinoma. Sci Rep. 2020;10:2757.

4. Pardoll DM. The blockade of immune checkpoints in cancer immunotherapy. Nat Rev Cancer. 2012;12:252264.

5. Legler DF, Loetscher M, Roos RS, Clark-Lewis I, Baggiolini M, Moser B. B cell-attracting chemokine 1, a human CXC chemokine expressed in lymphoid tissues, selectively attracts B lymphocytes via BLR1/CXCR5. J Exp Med. 1998;187:655-660. 
6. Cyster JG, Ansel KM, Reif K, Ekland EH, Hyman PL, Tang HL, Luther SA, Ngo VN. Follicular stromal cells and lymphocyte homing to follicles. Immunol Rev. 2000;176:181-193.

7. Ansel KM, Ngo VN, Hyman PL, Luther SA, Förster R, Sedgwick JD, Browning JL, Lipp M, Cyster JG. A chemokine-driven positive feedback loop organizes lymphoid follicles. Nature. 2000;406:309-314.

8. Shi K, Hayashida K, Kaneko M, Hashimoto J, Tomita T, Lipsky PE, Yoshikawa H, Ochi T. Lymphoid chemokine B cell-attracting chemokine-1 (CXCL13) is expressed in germinal center of ectopic lymphoid follicles within the synovium of chronic arthritis patients. J Immunol. 2001;166:650-655.

9. Gunn MD, Ngo VN, Ansel KM, Ekland EH, Cyster JG, Williams LT. A B-cell-homing chemokine made in lymphoid follicles activates Burkitt's lymphoma receptor-1. Nature. 1998;391:799-803.

10. Hussain M, Adah D, Tariq M, Lu Y, Zhang J, Liu J. CXCL13/CXCR5 signaling axis in cancer. Life Sci. 2019;227:175-186.

11. Airoldi I, Cocco C, Morandi F, Prigione I, Pistoia V. CXCR5 may be involved in the attraction of human metastatic neuroblastoma cells to the bone marrow. Cancer Immunol Immunother. 2008;57:541-548.

12. Biswas S, Sengupta S, Roy Chowdhury S, Jana S, Mandal G, Mandal PK, Saha N, Malhotra V, Gupta A, Kuprash DV et al. CXCL13-CXCR5 co-expression regulates epithelial to mesenchymal transition of breast cancer cells during lymph node metastasis. Breast Cancer Res Treat. 2014;143:265-276.

13. Ammirante M, Shalapour S, Kang Y, Jamieson CA, Karin M. Tissue injury and hypoxia promote malignant progression of prostate cancer by inducing CXCL13 expression in tumor myofibroblasts. Proc Natl Acad Sci U S A. 2014;111:14776-14781.

14. El Haibi CP, Sharma PK, Singh R, Johnson PR, Suttles J, Singh S, Lillard JW, Jr. PI3Kp110-, Src-, FAK-dependent and DOCK2-independent migration and invasion of CXCL13-stimulated prostate cancer cells. Mol Cancer. 2010;9:85.

15. Singh S, Singh R, Sharma PK, Singh UP, Rai SN, Chung LW, Cooper CR, Novakovic KR, Grizzle WE, Lillard JW, Jr. Serum CXCL13 positively correlates with prostatic disease, prostate-specific antigen and mediates prostate cancer cell invasion, integrin clustering and cell adhesion. Cancer Lett. 2009;283:29-35.

16. Ohtani H, Komeno T, Agatsuma Y, Kobayashi M, Noguchi M, Nakamura N. Follicular Dendritic Cell Meshwork in Angioimmunoblastic T-Cell Lymphoma Is Characterized by Accumulation of CXCL13(+) Cells. J Clin Exp Hematop. 2015;55:61-69.

17. Tang XF, Li GD, Li YL, Liang DN, Xia T, Zhou JY, Yao YQ, Wu WQ, Wang ZG, Yang YH et al. [Expressions of CXCL13, CD10 and bcl-6 in angioimmunoblastic T-cell lymphoma and peripheral T-cell lymphoma, not otherwise specified]. Zhonghua Bing Li Xue Za Zhi. 2009;38:224-230.

18. Yu H, Shahsafaei A, Dorfman DM. Germinal-center T-helper-cell markers PD-1 and CXCL13 are both expressed by neoplastic cells in angioimmunoblastic T-cell lymphoma. Am J Clin Pathol. 2009;131:33-41.

19. Yang M, Lu J, Zhang G, Wang Y, He M, Xu Q, Xu C, Liu H. CXCL13 shapes immunoactive tumor microenvironment and enhances the efficacy of PD-1 checkpoint blockade in high-grade serous ovarian cancer. J ImmunoTher Cancer. 2021;9.

20. Barone F, Nayar S, Campos J, Cloake T, Withers DR, Toellner KM, Zhang Y, Fouser L, Fisher B, Bowman S et al. IL-22 regulates lymphoid chemokine production and assembly of tertiary lymphoid organs. Proc Natl Acad Sci U S A. 2015;112:11024-11029.

21. Rhodes DR, Kalyana-Sundaram S, Mahavisno V, Varambally R, Yu J, Briggs BB, Barrette TR, Anstet MJ, Kincead-Beal C, Kulkarni $P$ et al. Oncomine 3.0: genes, pathways, and networks in a collection of 18,000 cancer 
gene expression profiles. Neoplasia. 2007;9:166-180.

22. Tang Z, Kang B, Li C, Chen T, Zhang Z. GEPIA2: an enhanced web server for large-scale expression profiling and interactive analysis. Nucleic Acids Res. 2019;47:W556-w560.

23. Xu S, Feng Y, Zhao S. Proteins with Evolutionarily Hypervariable Domains are Associated with Immune Response and Better Survival of Basal-like Breast Cancer Patients. Comput Struct Biotechnol J. 2019;17:430440.

24. Comprehensive molecular portraits of human breast tumours. Nature. 2012;490:61-70.

25. Nagy Á, Lánczky A, Menyhárt O, Győrffy B. Validation of miRNA prognostic power in hepatocellular carcinoma using expression data of independent datasets. Sci Rep. 2018;8:9227.

26. Wang Q, Xie L, Dang Y, Sun X, Xie T, Guo J, Han Y, Yan Z, Zhu W, Wang Y et al. OSIms: A Web Server to Evaluate the Prognostic Value of Genes in Leiomyosarcoma. Front Oncol. 2019;9:190.

27. Wang Q, Wang F, Lv J, Xin J, Xie L, Zhu W, Tang Y, Li Y, Zhao X, Wang Y et al. Interactive online consensus survival tool for esophageal squamous cell carcinoma prognosis analysis. Oncol Lett. 2019;18:1199-1206.

28. Xie L, Wang Q, Dang Y, Ge L, Sun X, Li N, Han Y, Yan Z, Zhang L, Li Y et al. OSkirc: a web tool for identifying prognostic biomarkers in kidney renal clear cell carcinoma. Future Oncol. 2019;15:3103-3110.

29. Zhang G, Wang Q, Yang M, Yuan Q, Dang Y, Sun X, An Y, Dong H, Xie L, Zhu W et al. OSblca: A Web Server for Investigating Prognostic Biomarkers of Bladder Cancer Patients. Front Oncol. 2019;9:466.

30. Wang Q, Zhang L, Yan Z, Xie L, An Y, Li H, Han Y, Zhang G, Dong H, Zheng H et al. OScc: an online survival analysis web server to evaluate the prognostic value of biomarkers in cervical cancer. Future Oncol. 2019;15:3693-3699.

31. Yan Z, Wang Q, Sun X, Ban B, Lu Z, Dang Y, Xie L, Zhang L, Li Y, Zhu W et al. OSbrca: A Web Server for Breast Cancer Prognostic Biomarker Investigation With Massive Data From Tens of Cohorts. Front Oncol. 2019;9:1349.

32. Xie L, Wang Q, Nan F, Ge L, Dang Y, Sun X, Li N, Dong H, Han Y, Zhang G et al. OSacc: Gene Expression-Based Survival Analysis Web Tool For Adrenocortical Carcinoma. Cancer Manag Res. 2019;11:9145-9152.

33. Wang F, Wang Q, Li N, Ge L, Yang M, An Y, Zhang G, Dong H, Ji S, Zhu W et al. OSuvm: An interactive online consensus survival tool for uveal melanoma prognosis analysis. Mol Carcinog. 2020;59:56-61.

34. Ru B, Wong CN, Tong Y, Zhong JY, Zhong SSW, Wu WC, Chu KC, Wong CY, Lau CY, Chen I et al. TISIDB: an integrated repository portal for tumor-immune system interactions. Bioinformatics. 2019;35:4200-4202.

35. Li T, Fan J, Wang B, Traugh N, Chen Q, Liu JS, Li B, Liu XS. TIMER: A Web Server for Comprehensive Analysis of Tumor-Infiltrating Immune Cells. Cancer Res. 2017;77:e108-e110.

36. Li B, Li JZ. A general framework for analyzing tumor subclonality using SNP array and DNA sequencing data. Genome Biol. 2014;15:473.

37. Mermel CH, Schumacher SE, Hill B, Meyerson ML, Beroukhim R, Getz G. GISTIC2.0 facilitates sensitive and confident localization of the targets of focal somatic copy-number alteration in human cancers. Genome Biol. 2011;12:R41.

38. Danaher P, Warren S, Dennis L, D'Amico L, White A, Disis ML, Geller MA, Odunsi K, Beechem J, Fling SP. Gene expression markers of Tumor Infiltrating Leukocytes. J ImmunoTher Cancer. 2017;5:18.

39. Bhattacharya S, Dunn P, Thomas CG, Smith B, Schaefer H, Chen J, Hu Z, Zalocusky KA, Shankar RD, Shen-Orr SS et al. ImmPort, toward repurposing of open access immunological assay data for translational and clinical research. Sci Data. 2018;5:180015.

Page 25/33 
40. Nirmal AJ, Regan T, Shih BB, Hume DA, Sims AH, Freeman TC. Immune Cell Gene Signatures for Profiling the Microenvironment of Solid Tumors. Cancer Immunol Res. 2018;6:1388-1400.

41. Guo X, Zhang Y, Zheng L, Zheng C, Song J, Zhang Q, Kang B, Liu Z, Jin L, Xing R et al. Global characterization of T cells in non-small-cell lung cancer by single-cell sequencing. Nat Med. 2018;24:978-985.

42. Uhlén M, Fagerberg L, Hallström BM, Lindskog C, Oksvold P, Mardinoglu A, Sivertsson Å, Kampf C, Sjöstedt E, Asplund A et al. Proteomics. Tissue-based map of the human proteome. Science. 2015;347:1260419.

43. Vasaikar SV, Straub P, Wang J, Zhang B. LinkedOmics: analyzing multi-omics data within and across 32 cancer types. Nucleic Acids Res. 2018;46:D956-d963.

44. Sticht C, De La Torre C, Parveen A, Gretz N. miRWalk: An online resource for prediction of microRNA binding sites. PLoS One. 2018;13:e0206239.

45. Ravelli A, Roviello G, Cretella D, Cavazzoni A, Biondi A, Cappelletti MR, Zanotti L, Ferrero G, Ungari M, Zanconati F et al. Tumor-infiltrating lymphocytes and breast cancer: Beyond the prognostic and predictive utility. Tumour Biol. 2017;39:1010428317695023.

46. Azimi F, Scolyer RA, Rumcheva P, Moncrieff M, Murali R, McCarthy SW, Saw RP, Thompson JF. Tumorinfiltrating lymphocyte grade is an independent predictor of sentinel lymph node status and survival in patients with cutaneous melanoma. J Clin Oncol. 2012;30:2678-2683.

47. Ohtani H. Focus on TILs: prognostic significance of tumor infiltrating lymphocytes in human colorectal cancer. Cancer Immun. 2007;7:4.

48. Sawant A, Hensel JA, Chanda D, Harris BA, Siegal GP, Maheshwari A, Ponnazhagan S. Depletion of plasmacytoid dendritic cells inhibits tumor growth and prevents bone metastasis of breast cancer cells. J Immunol. 2012;189:4258-4265.

49. Konecny GE, Wang C, Hamidi H, Winterhoff B, Kalli KR, Dering J, Ginther C, Chen H-W, Dowdy S, Cliby W. Prognostic and therapeutic relevance of molecular subtypes in high-grade serous ovarian cancer. JNCl: Journal of the national cancer institute. 2014;106.

50. Peng D, Kryczek I, Nagarsheth N, Zhao L, Wei S, Wang W, Sun Y, Zhao E, Vatan L, Szeliga W et al. Epigenetic silencing of TH1-type chemokines shapes tumour immunity and immunotherapy. Nature. 2015;527:249-253.

51. Qu QX, Xie F, Huang Q, Zhang XG. Membranous and Cytoplasmic Expression of PD-L1 in Ovarian Cancer Cells. Cell Physiol Biochem. 2017;43:1893-1906.

52. Zeng Y, Li B, Liang Y, Reeves PM, Qu X, Ran C, Liu Q, Callahan MV, Sluder AE, Gelfand JA et al. Dual blockade of CXCL12-CXCR4 and PD-1-PD-L1 pathways prolongs survival of ovarian tumor-bearing mice by prevention of immunosuppression in the tumor microenvironment. FASEB J. 2019;33:6596-6608.

53. Zhu Y, Yang J, Xu D, Gao XM, Zhang Z, Hsu JL, Li CW, Lim SO, Sheng YY, Zhang Y et al. Disruption of tumourassociated macrophage trafficking by the osteopontin-induced colony-stimulating factor-1 signalling sensitises hepatocellular carcinoma to anti-PD-L1 blockade. Gut. 2019;68:1653-1666.

54. Wherry EJ. T cell exhaustion. Nat Immunol. 2011;12:492-499.

55. Wherry EJ, Kurachi M. Molecular and cellular insights into T cell exhaustion. Nat Rev Immunol. 2015;15:486499.

56. Zarour HM. Reversing T-cell Dysfunction and Exhaustion in Cancer. Clin Cancer Res. 2016;22:1856-1864.

57. Ribas A, Wolchok JD. Cancer immunotherapy using checkpoint blockade. Science. 2018;359:1350-1355.

58. Anderson AC, Joller N, Kuchroo VK. Lag-3, Tim-3, and TIGIT: Co-inhibitory Receptors with Specialized Functions in Immune Regulation. Immunity. 2016;44:989-1004. 
59. Dudeck A, Köberle M, Goldmann O, Meyer N, Dudeck J, Lemmens S, Rohde M, Roldán NG, Dietze-Schwonberg K, Orinska Z et al. Mast cells as protectors of health. J Allergy Clin Immunol. 2019;144:S4-s18.

60. Eissmann MF, Dijkstra C, Jarnicki A, Phesse T, Brunnberg J, Poh AR, Etemadi N, Tsantikos E, Thiem S, Huntington ND et al. IL-33-mediated mast cell activation promotes gastric cancer through macrophage mobilization. Nat Commun. 2019;10:2735.

61. Komi DEA, Redegeld FA. Role of Mast Cells in Shaping the Tumor Microenvironment. Clin Rev Allergy Immunol. 2020;58:313-325.

\section{Figures}

\section{A $\quad$ CXCL13}
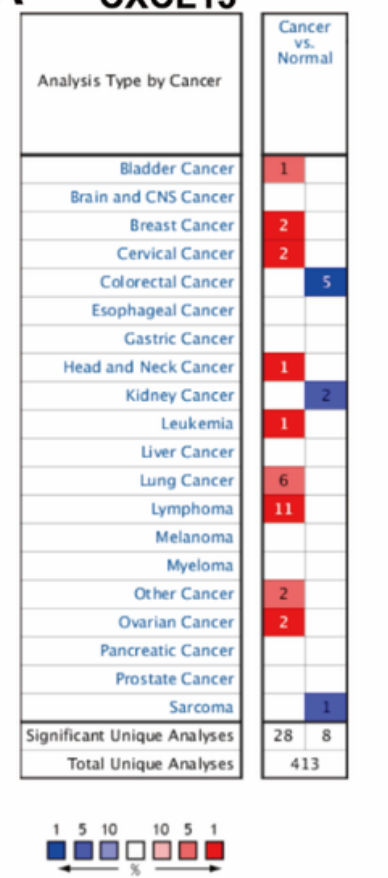

Gene rank percentile(\%)
B

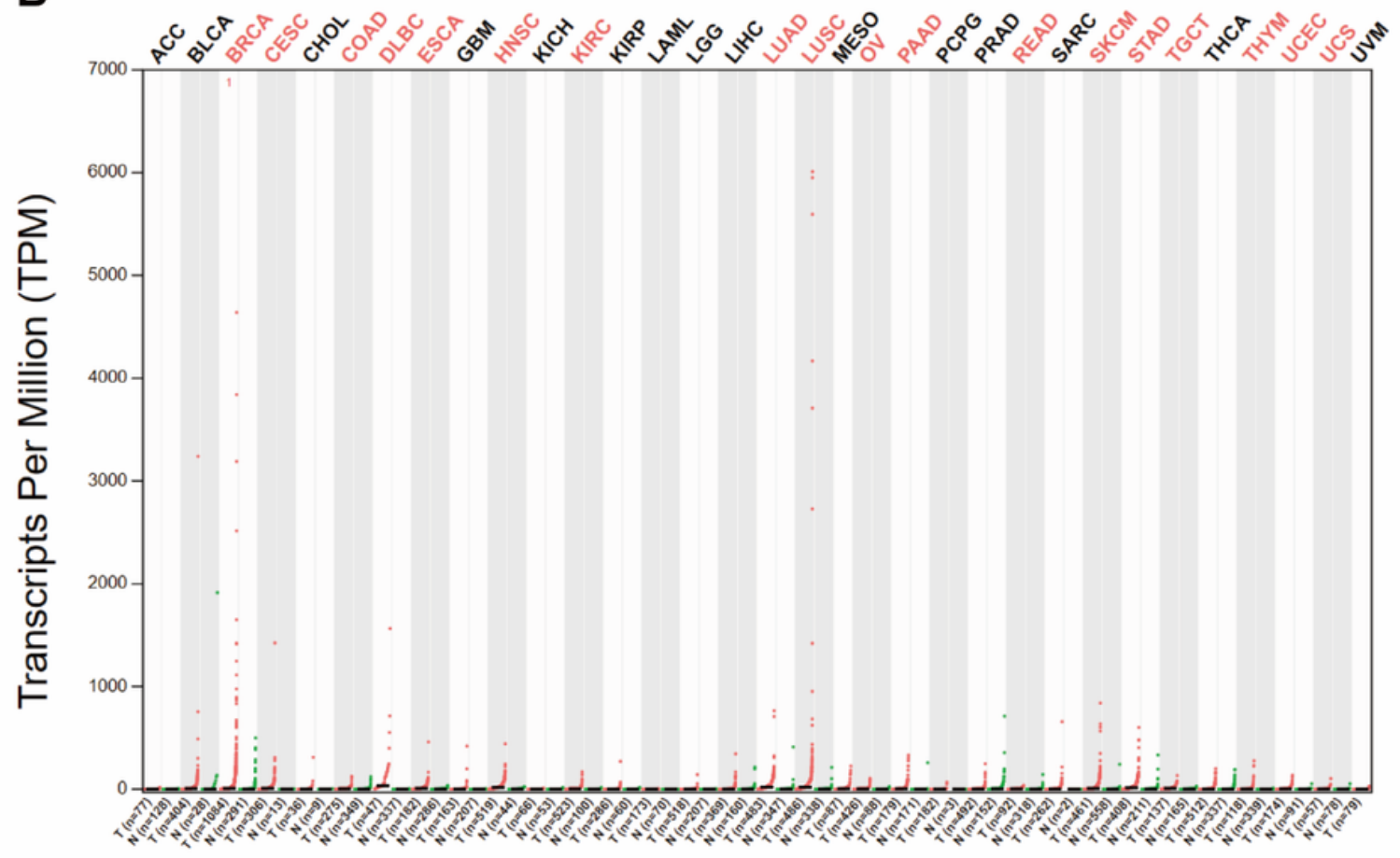

\section{Figure 1}

CXCL13 expression within diverse human cancer types. (A) CXCL13 up-regulation or down-regulation within diverse cancer datasets relative to non-carcinoma samples from Oncomine database. (B) CXCL13 expression within TCGAderived cancers was measured through GEPIA2. The dots stand for expression in individual cancer or noncarcinoma samples. 
A Ovarian Cancer os CXCL13 (205242_at)

B Ovarian Cancer PFS C

C Lung Cancer OS

D Lung Cancer PFS
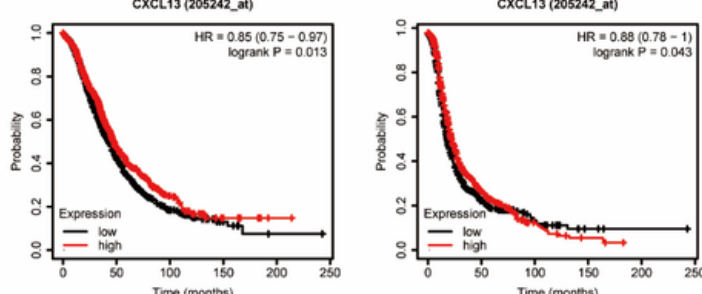

CXCL13 (205242_at)
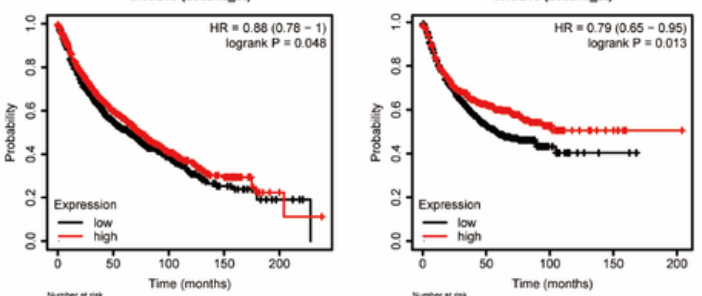

E Gastric Cancer OS

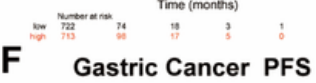

G Breast Cancer OS
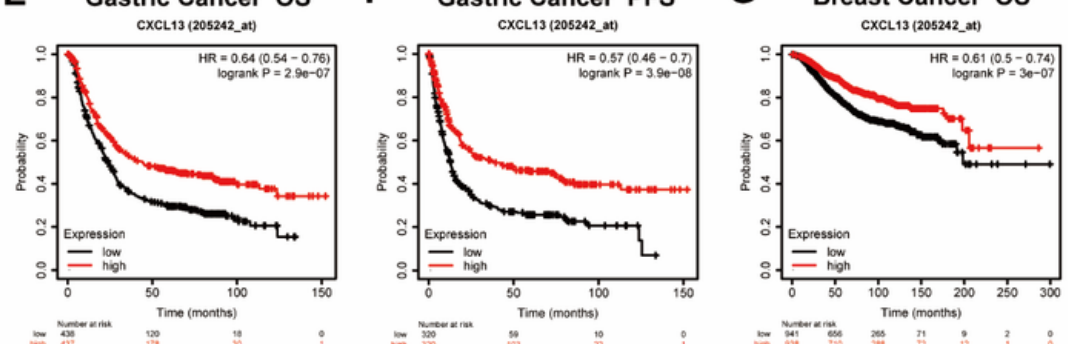

H Breast Cancer RFS
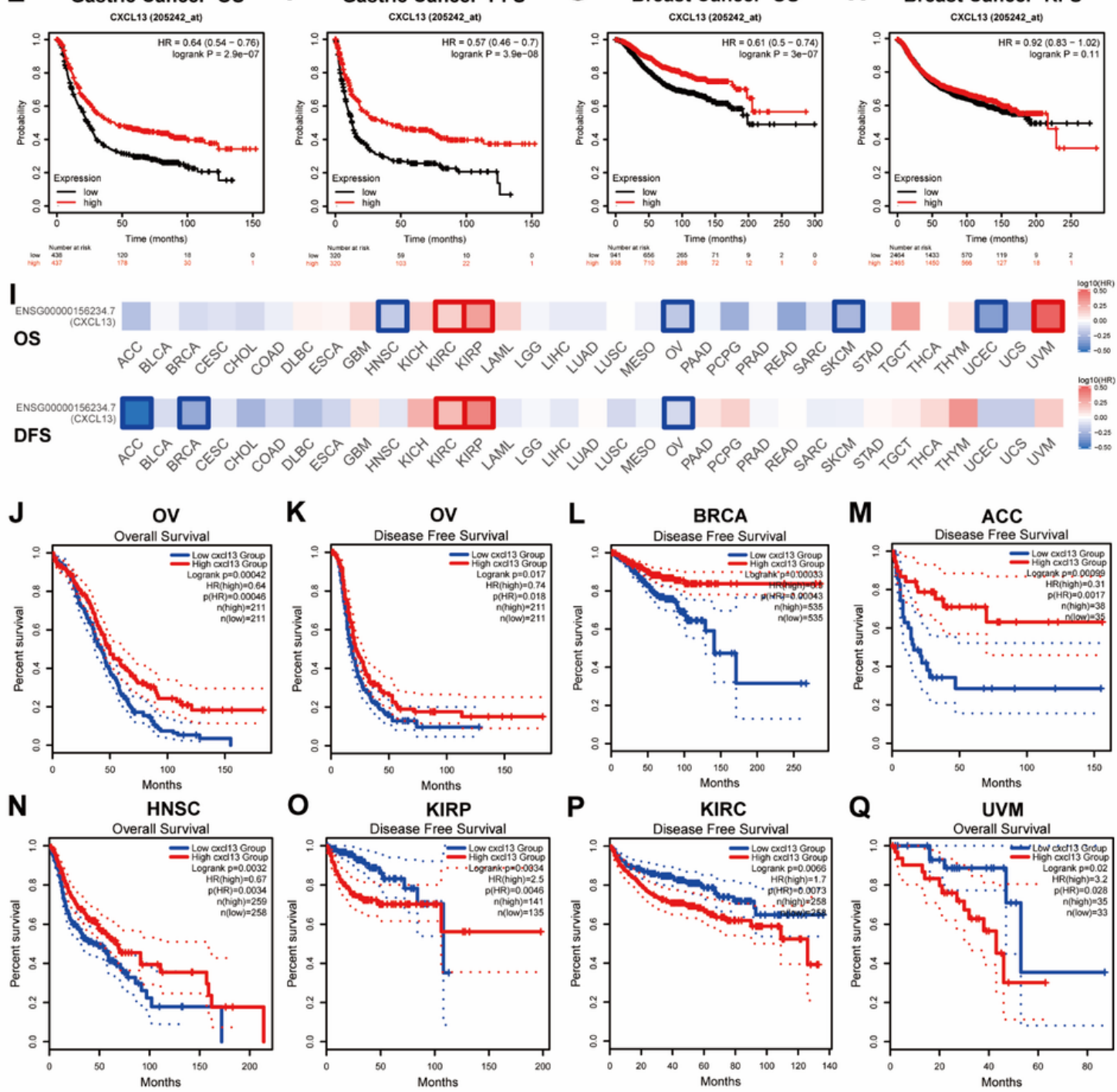

Figure 2

Significance of CXCL13 in predicting the prognosis of diverse cancers assessed through Kaplan-Meier Plotter(A-H) and GEPIA2 (I-Q). OS and PFS curves in (A, B) ovarian cancer $(n=1656, n=1435),(C, D)$ lung cancer $(n=1925, n=$ 982), and $(E, F)$ gastric cancer $(n=875, n=640)$. OS and RFS curves in $(G, H)$ breast cancer $(n=1879, n=4929)$. (I) Heat map of CXCL13 in 33 types of TCGA tumors. The heat map reveals HRs in logarithmic scale (log10) for CXCL13. The blue and red blocks suggest lower and higher risks, respectively. Rectangles with frames indicate statistically significant findings in the prognostic analysis. OS and DFS curves in $(\mathrm{J}, \mathrm{K})$ OV $(\mathrm{n}=422)$, OS curves in (N) HNSC ( $n=517)$ and (Q) UVM ( $n=68)$. DFS curves in (L) BRCA ( $n=1070),(M)$ ACC $(n=73),(0) \operatorname{KIRP}(n=276)$ and $(P) \operatorname{KIRC}(\mathrm{n}=516)$. 
A

B
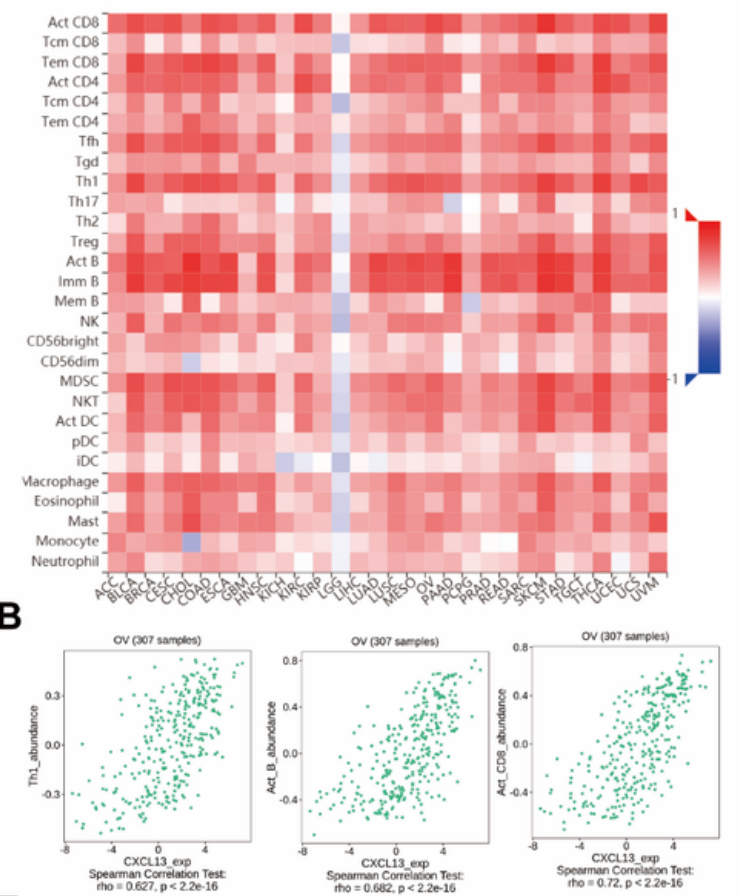

E

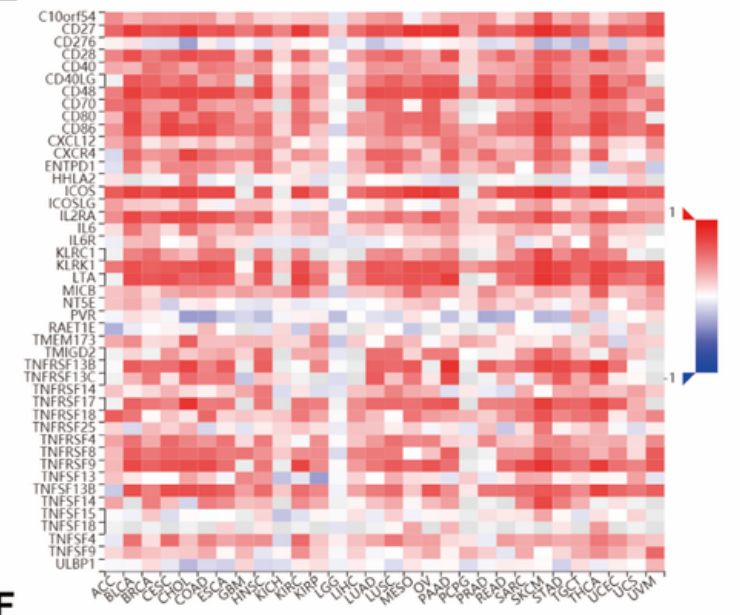

$\mathbf{F}$
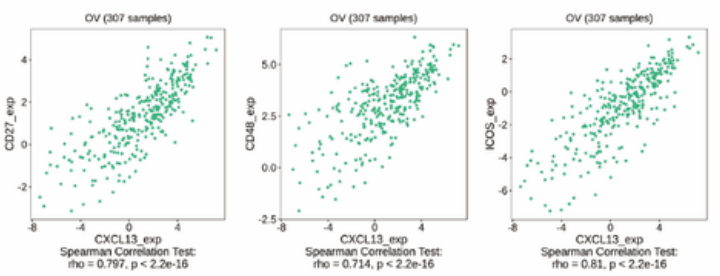

C

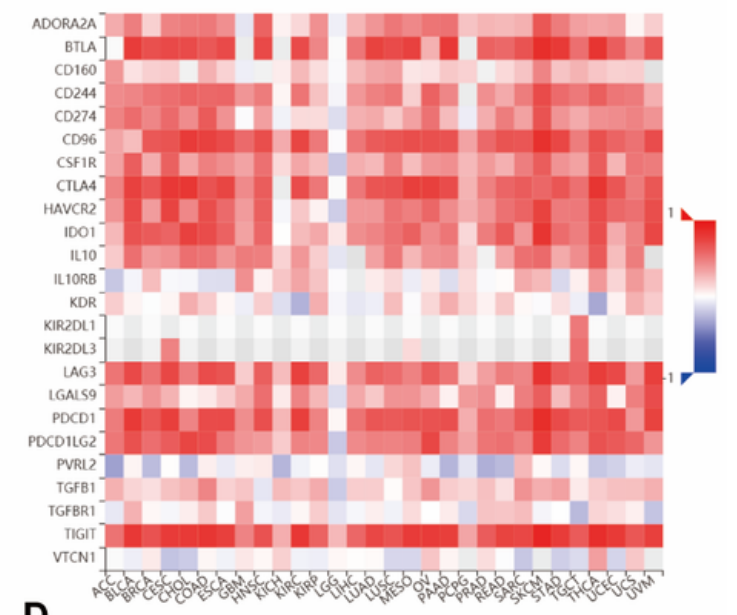

D
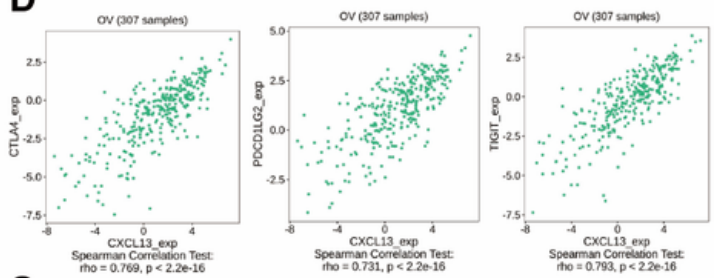

G
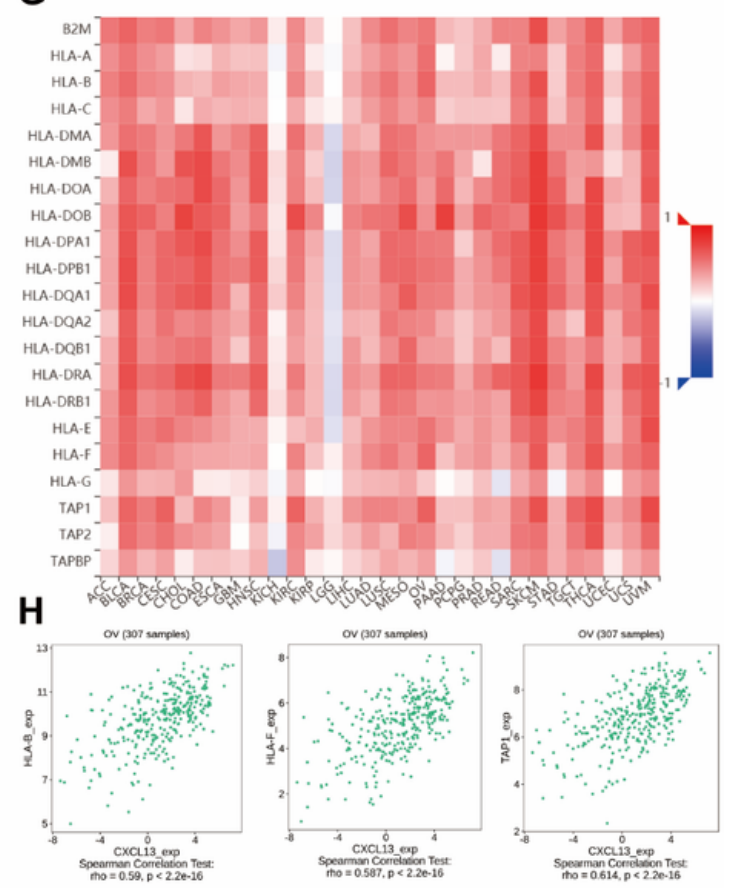

Figure 3

Spearman's correlation between CXCL13 and immunomodulators as well as lymphocytes (TISIDB). (A) Associations of TILs abundances with CXCL13 level. (B) The 3 most significant TILs with the highest Spearman's correlation with CXCL13 level. (C) Associations of immunoinhibitors abundances with CXCL13 level. (D) The 3 most significant immunoinhibitors with the highest Spearman's correlation with CXCL13 level. (E) Associations of immunostimulators abundances with CXCL13 level. (F) The 3 most significant immunostimulators with the highest Spearman's correlation with CXCL13 level. (G) Associations of MHC molecules with CXCL13 level. (H) The 3 most significant MHC molecules with the highest Spearman's correlation with CXCL13 level. Blue and red cells stand for negative and positive correlations, separately. Color intensity is in direct proportion to correlation strength. MHC major histocompatibility complex and TILs tumor-infltrating lymphocytes. 
A
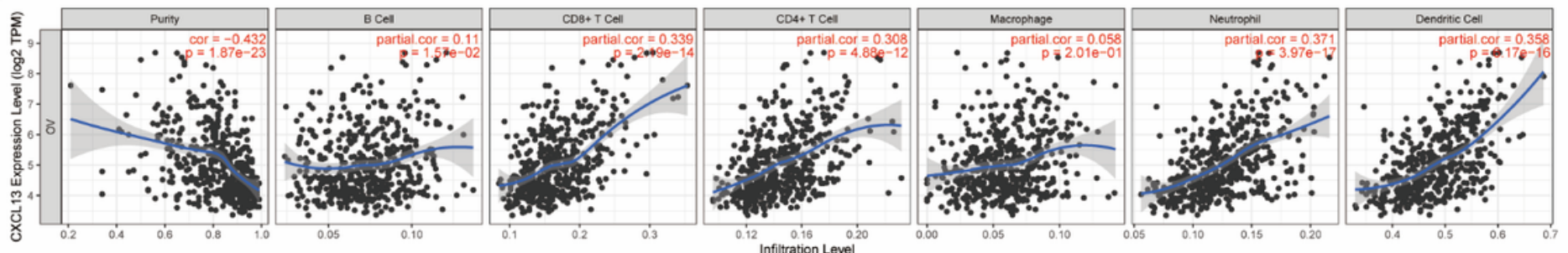

B

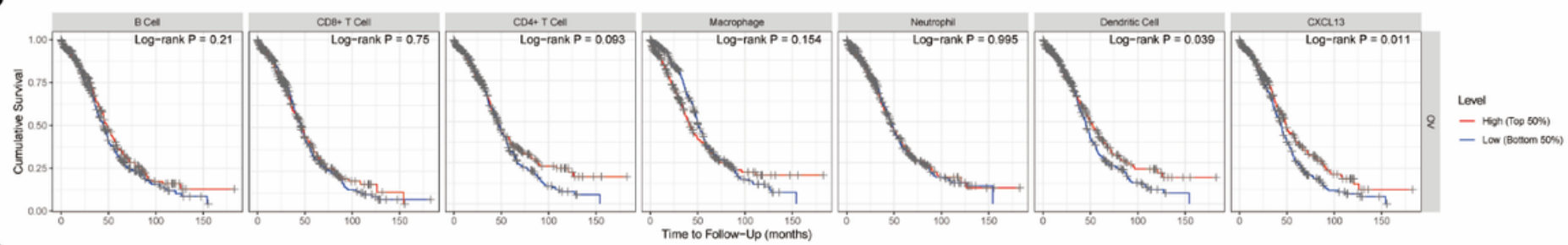

C

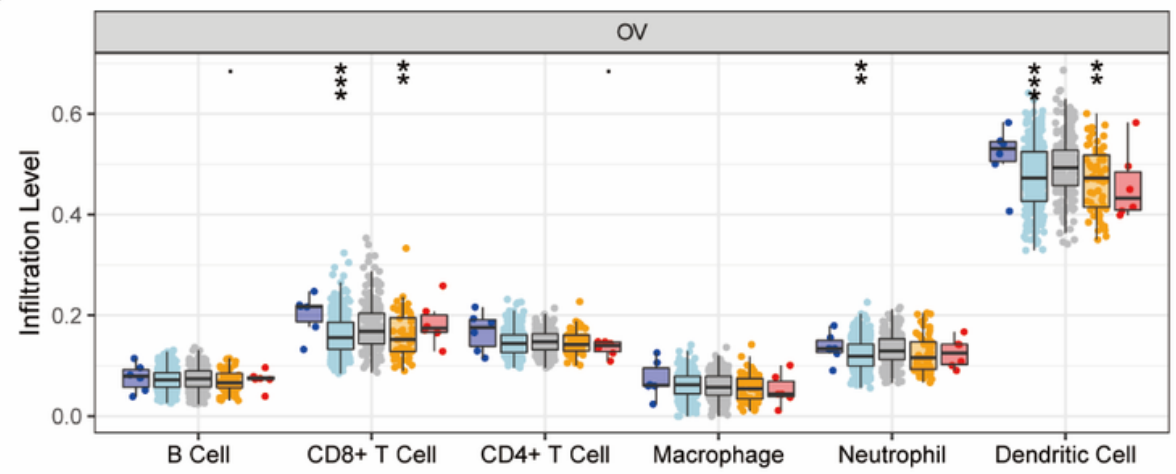

Copy Number

审 Deep Deletion

审 Arm-level Deletion

审 Diploid/Normal

审 Arm-level Gain

审 High Amplication

\section{Figure 4}

Associations of CXCL13 with immune infiltration degrees within OV. (A) Associations of CXCL13 level with immune infiltration degrees of CD8+ T cells, CD4+ T cells, B cells, neutrophils, DCs, and tumor purity. (B) Kaplan-Meier curves regarding immune infiltration degrees and CXCL13 level within OV. (C) Tumor-infiltration degrees of OV showing diverse SCNAs defined through GISTIC 2.0, which included high amplification (2), arm-level gain (1), diploid/normal

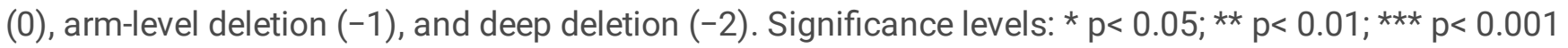


A

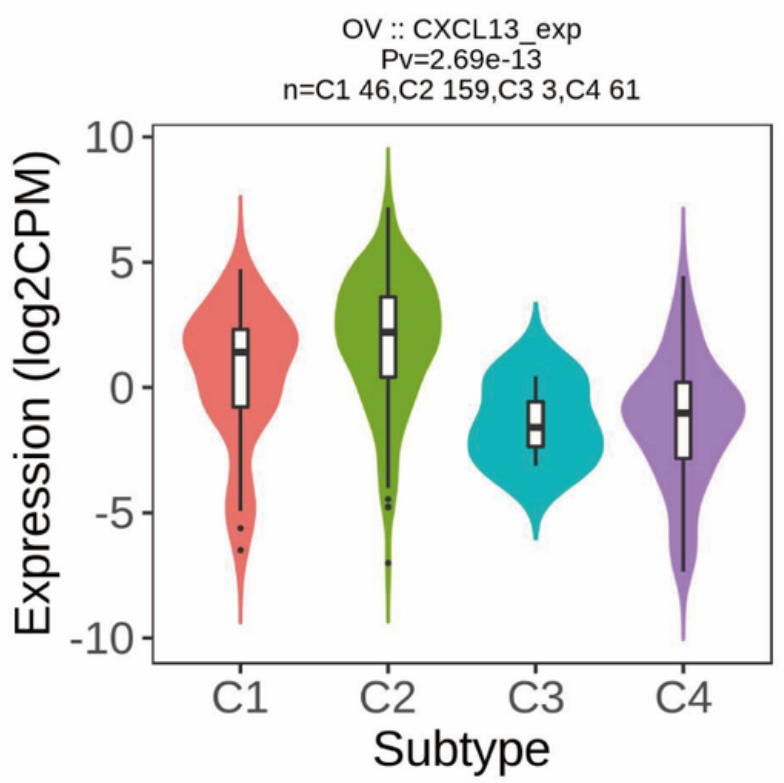

B

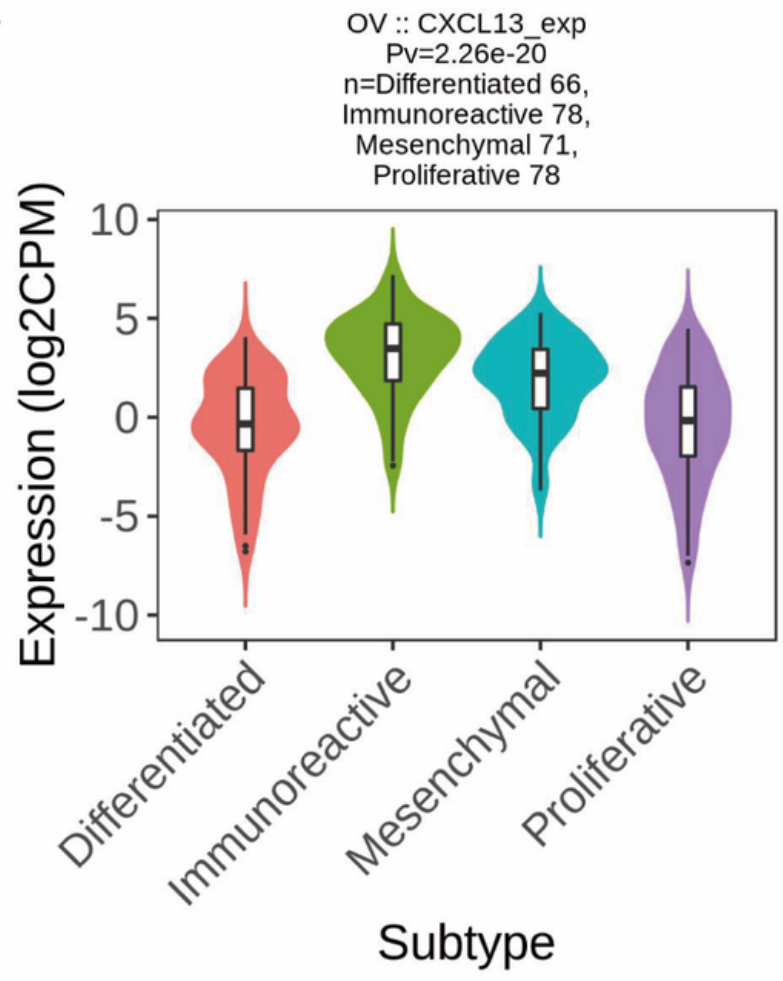

C



Tumor

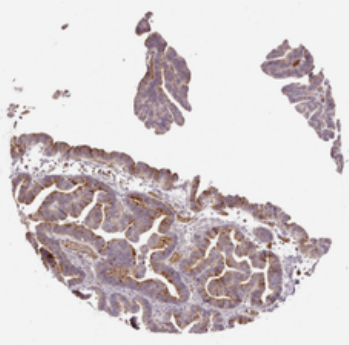

Normal

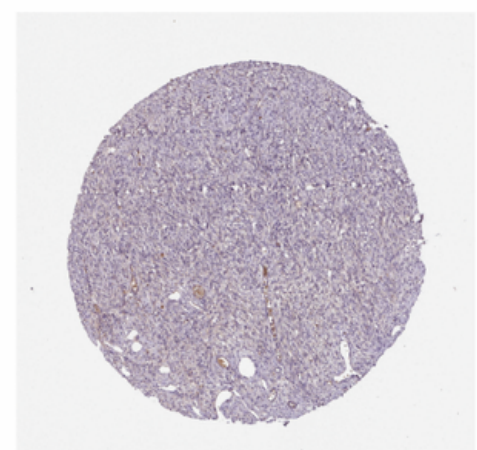

Figure 5

CXCL13 level within OV. (A) CXCL13 expression within diverse TISIDB-derived OV immune subtypes. (B) CXCL13 expression within diverse TISIDB-derived OV molecular subtypes. (C) CXCL13 expression within diverse stages of GEPIA2-derived OV. CXCL13 gene expression data were calculated relative to log counts per million mapped reads $(\log 2 \mathrm{CPM})$ in (A, B) and log2(TPM + 1) in (C). (D) Immunohistochemistry of CXCL13 based on the Human Protein Atlas. CXCL13 in normal tissue: https://www.proteinatlas.org/ENSG00000156234-CXCL13/tissue/ovary\# img; CXCL13 in tumor tissue: https://www.proteinatlas.org/ENSG00000156234-CXCL13/pathology/ ovarian+cancer\#img. Tumor: CXCL13 protein expression within cancer samples (medium, staining, moderate staining intensity, $>75 \%$ quantity); Normal: CXCL13 protein expression within non-carcinoma samples (no staining, negative staining intensity, and no quantity detected). 


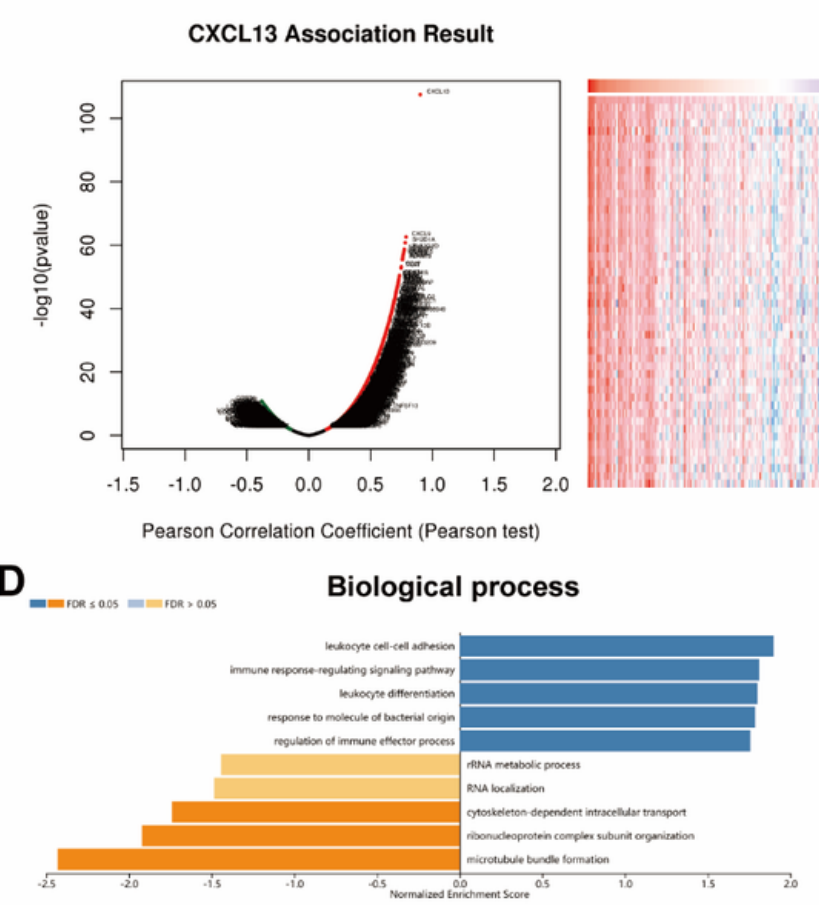

E

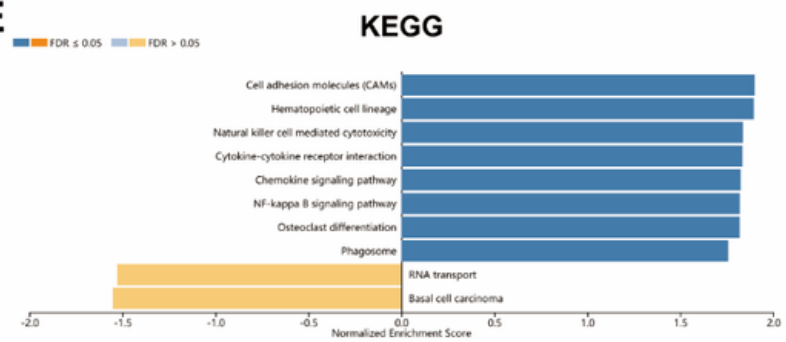

F

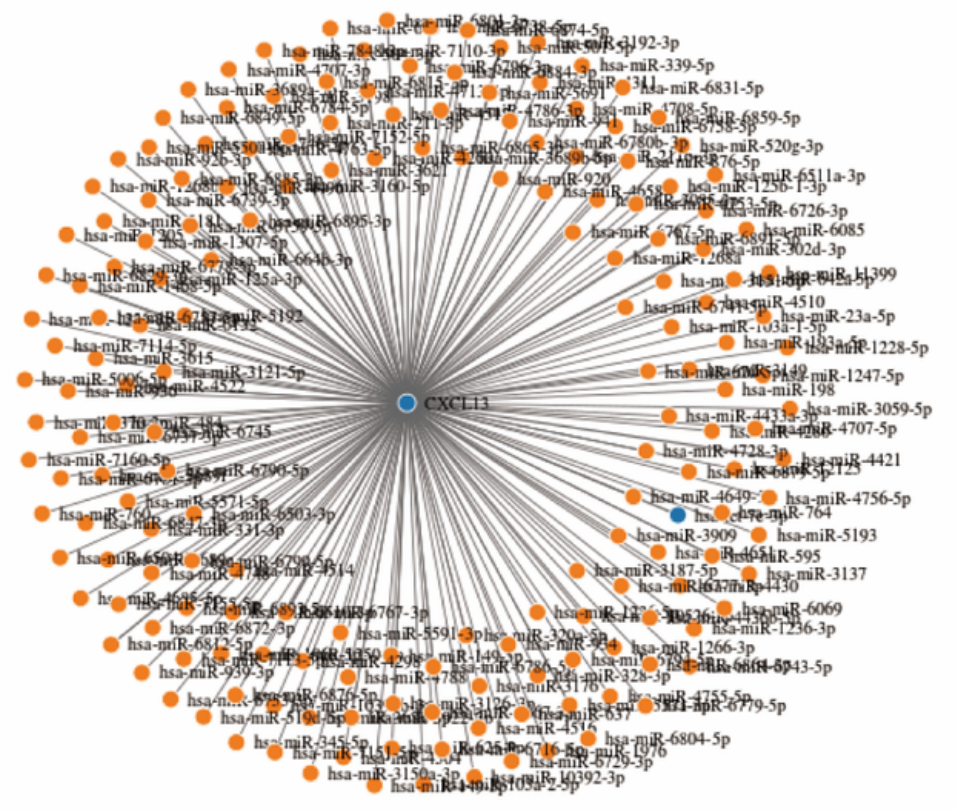

\section{Top20 miRNA}

hsa-miR-550a-5p

hsa-miR-3688-5p

hsa-miR-4512

hsa-miR-4728-3p

hsa-miR-5193

hsa-miR-6738-5p

hsa-miR-6812-5p

hsa-miR-1226-3p

hsa-miR-520g-3p

hsa-miR-6510-3p

hsa-miR-2116-3p

hsa-miR-6799-5p

hsa-miR-320a-5p

hsa-miR-6801-3p

hsa-let-7e-3p

hsa-let-7e-3p

hsa-miR-23a-5p

hsa-miR-103a-2-5p

hsa-miR-103a-1-5p

hsa-miR-196a-5p

Figure 6

CXCL13 co-expression genes within OV (LinkedOmics). (A) The CXCL13-related genes within OV were discovered by Pearson test. Green and red dots indicate genes with significant negative and positive correlations with CXCL13, separately. (B, C) Heatmaps displaying the 50 most significant CXCL13-related genes within OV (both positive and negative). (D, E) Markedly associated GO: BP annotations together with KEGG pathway analysis for CXCL13 within OV. (F) CXCL13 and its predicted miRNAs (yellow and blue circles stand for targeted miRNAs and CXCL13, respectively). The interaction between the CXCL13 and related miRNAs is shown in the form of lines). GO: Gene Ontology; FDR: false discovery rate; KEGG: Kyoto Encyclopedia of Genes and Genomes.

\section{Supplementary Files}


This is a list of supplementary files associated with this preprint. Click to download.

- HonglanQuMSSupplementarymaterial.docx 